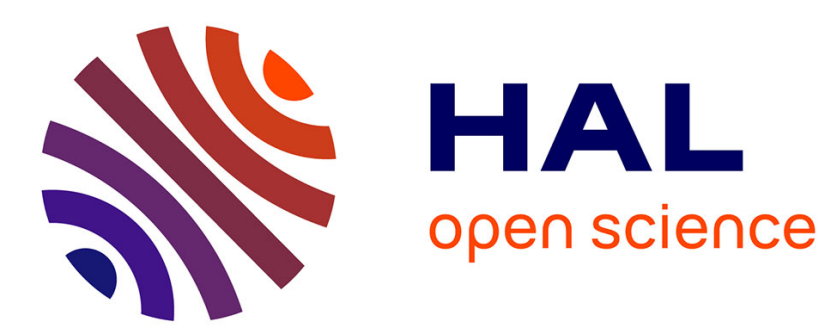

\title{
On subadditive theorems for group actions and homogenization
}

\author{
Omar Anza Hafsa, Jean-Philippe Mandallena
}

\section{To cite this version:}

Omar Anza Hafsa, Jean-Philippe Mandallena. On subadditive theorems for group actions and homogenization. Bulletin des Sciences Mathématiques, 2020, 158, pp.102821. 10.1016/j.bulsci.2019.102821 . hal-02512875

\section{HAL Id: hal-02512875 \\ https://hal.science/hal-02512875}

Submitted on 31 May 2021

HAL is a multi-disciplinary open access archive for the deposit and dissemination of scientific research documents, whether they are published or not. The documents may come from teaching and research institutions in France or abroad, or from public or private research centers.
L'archive ouverte pluridisciplinaire HAL, est destinée au dépôt et à la diffusion de documents scientifiques de niveau recherche, publiés ou non, émanant des établissements d'enseignement et de recherche français ou étrangers, des laboratoires publics ou privés. 


\title{
On subadditive theorems for group actions and homogenization
}

\author{
Omar Anza Hafsa, Jean-Philippe Mandallena* \\ Universite de Nimes, Laboratoire MIPA, Site des Carmes, Place Gabriel Péri, \\ 30021 Nimes, France
}

\section{A B S T R A C T}
We prove subadditive theorems à la Akcoglu-Krengel on mea- sure spaces with acting groups. Applications to homogeniza- tion of nonconvex integrals in Cheeger-Sobolev spaces are also developed.

\section{Introduction}

Let $(X, \mathcal{M}, \mu)$ be a measure space, let $\mathbb{G}$ be a group acting right measurably ${ }^{1}$ on $X$ such that $\mu$ is $\mathbb{G}$-invariant. Let $\mathcal{B} \subset \mathcal{M}$ be a ring which is right stable by $\mathbb{G}$ and let

$$
\mathcal{S}: \mathcal{B} \rightarrow L^{1}(\Omega, \mathcal{F}, \mathbb{P}) \quad(\text { resp. } \mathcal{S}: \mathcal{B} \rightarrow \mathbb{R})
$$

\footnotetext{
* Corresponding author.

E-mail addresses: omar.anza-hafsa@unimes.fr (O. Anza Hafsa), jean-philippe.mandallena@unimes.fr (J.-P. Mandallena).

1 By a group $\mathbb{G}$ acting right measurably on the measure space $(X, \mathcal{M}, \mu)$, we mean that there is a binary operation $\Phi: X \times \mathbb{G} \rightarrow X,(x, g) \mapsto \Phi(x, g)=: x g$, such that for each $g \in \mathbb{G}$, the map $\Phi(\cdot, g)$ is $(\mathcal{M}, \mathcal{M})$ -
} 
be subadditive and $\mathbb{G}$-covariant (resp. $\mathbb{G}$-invariant), where $\left(\Omega, \mathcal{F}, \mathbb{P},\left\{\tau_{g}\right\}_{g \in \mathbb{G}}\right)$ is a measurable dynamical $\mathbb{G}$-system, and let $\left\{J_{n}\right\}_{n \in \mathbb{N}}$ be a sequence of sets of $\mathcal{B}$. In this paper we are concerned with the problem of characterizing the following limit

$$
\left.\lim _{n \rightarrow \infty} \frac{\mathcal{S}\left(J_{n}\right)(\omega)}{\mu\left(J_{n}\right)} \text { for P-a.a. } \omega \in \Omega \quad \text { (resp. } \lim _{n \rightarrow \infty} \frac{\mathcal{S}\left(J_{n}\right)}{\mu\left(J_{n}\right)}\right) .
$$

Such limit problems are of interest for the development of homogenization of integrals of the calculus of variations in the setting of Cheeger-Sobolev spaces (see [2]). Other motivations can be found in the study of percolation theory (see [23]).

Motivated by problems of statistical mechanics, additive theorems were first proved in 1931 by von Neumann (see [33]) and Birkhoff (see [5]) in the context of measure preserving $\mathbb{Z}$-actions. Then, in 1972 , Tempelman generalized these results to the multidimensional case in the context of measure $\mathbb{Z}^{N}$-actions (see [40]) and Nguyen and Zessin [35]) but also to the setting of amenable semi-groups (see [41] and [27, Theorem 4.4]). Later, in 1999, the theorems of von Neumann and Birkhoff were extended by Lindenstrauss to the setting of amenable groups under weaker conditions than those of Templeman (see [28,29] and also Calderon [7]).

Beside this, motivated by the study of percolation theory, subadditive theorems were proved, in the context of measure preserving $\mathbb{Z}^{N}$-actions, first in 1968 by Kingman in the unidimensional case (see [25,26]) and then in 1981 by Akcoglu and Krengel in the multidimensional case (see [3,27] and Derriennic [10], Smythe [39], Nguyen [34]). In 2014, Dooley, Golodets and Zhang extended Kingman's theorem to the setting of countable discrete amenable groups (see [12] and [16]). In this paper, we extend Akcoglu-Krengel's theorem to the setting of measure spaces with acting groups.

Multidimensional subadditive theorems à la Akcoglu-Krengel were adapted first in 1986 by Dal Maso and Modica for dealing with homogenization of convex integral functionals of the calculus of variations defined on Sobolev spaces (see $[14,15]$ ) and then in 1994 by Messaoudi and Michaille for studying nonconvex homogenization problems (see $[31,30])$. In the same spirit, the object of this paper is to establish subadditive theorems allowing to deal with nonconvex homogenization problems in Cheeger-Sobolev spaces.

The plan of the paper is as follows. In Sect. 2, we state our subadditive theorems: the main new result is Theorem 2.14 whose proof uses Lindenstrauss's ergodic theorem (see [29]) and a maximal inequality established in [12]. To obtain such subadditive results we need to introduce a notion of meshability with respect to the acting group: this is defined in $\$ 2.1$. Finally, to illustrate our results, applications to homogenization of nonconvex integrals in Cheeger-Sobolev spaces are developed in Sect. 3.

\section{Subadditive theorems}

Let $(X, \mathcal{M}, \mu)$ be a measure space, let $\mathbb{G}$ be a group acting right measurably on $X$ such that $\mu$ is $\mathbb{G}$-invariant, i.e. 


$$
\mu(A g)=\mu(A) \text { for all } g \in \mathbb{G} \text { and all } A \in \mathcal{M}
$$

with $A g:=\{a g: a \in A\}$. Let $\mathcal{B}$ be a subclass of sets $B \in \mathcal{M}$ such that $\mu(B)<\infty$. For each $A \in \mathcal{M}$, we consider the class $\mathcal{D}(A)$ defined by

$$
\mathcal{D}(A):=\left\{H \subset \mathbb{G}:\{A g\}_{g \in H} \text { is disjoint }\right\} .
$$

In what follows, $|\cdot|$ denotes the counting measure on $\mathbb{G}$ and, for any $H \subset \mathbb{G}, \mathcal{P}_{\mathrm{f}}(H)$ denotes the class of finite subsets of $H$.

\subsection{A subadditive lemma}

We need the following notion of meshability.

Definition 2.1. The measure space $(X, \mathcal{M}, \mu)$ is said to be $\mathbb{G}$-meshable if there is $\left\{\mathbb{U}_{k}\right\}_{k \in \mathbb{N}^{*}} \subset \mathcal{B}$ with $\mu\left(\mathbb{U}_{k}\right)>0$ for all $k \in \mathbb{N}^{*}$, such that for each $k \in \mathbb{N}^{*}$ there exists $\mathbb{G}_{k} \in \mathcal{D}\left(\mathbb{U}_{k}\right)$ with the property that for each $q \in \mathbb{N}^{*}$ there exist $G_{q, k}^{-} \subset G_{q, k}^{+} \in \mathcal{P}_{\mathrm{f}}\left(\mathbb{G}_{k}\right)$ such that:

$$
\begin{gathered}
\cup_{g \in G_{q, k}^{-}} \mathbb{U}_{k} g \subset \mathbb{U}_{q} \subset \bigcup_{g \in G_{q, k}^{+}} \mathbb{U}_{k} g \\
\lim _{q \rightarrow \infty} \frac{\mu\left(\cup_{g \in G_{q, k}^{+}} \mathbb{U}_{k} g \backslash \cup_{g \in G_{q, k}^{-}} \mathbb{U}_{k} g\right)}{\mu\left(\mathbb{U}_{q}\right)}=\lim _{q \rightarrow \infty} \frac{\mu\left(\cup_{g \in G_{q, k}^{+} \backslash G_{q, k}^{-}} \mathbb{U}_{k} g\right)}{\mu\left(\mathbb{U}_{q}\right)} \\
=\lim _{q \rightarrow \infty}\left|G_{q, k}^{+} \backslash G_{q, k}^{-}\right| \frac{\mu\left(\mathbb{U}_{k}\right)}{\mu\left(\mathbb{U}_{q}\right)}=0 .
\end{gathered}
$$

The quadruple $\left(\left\{\mathbb{U}_{k}\right\}_{k \in \mathbb{N}^{*}},\left\{\mathbb{G}_{k}\right\}_{k \in \mathbb{N}^{*}},\left\{G_{q, k}^{-}\right\}_{(q, k) \in \mathbb{N}^{*} \times \mathbb{N}^{*}},\left\{G_{q, k}^{+}\right\}_{(q, k) \in \mathbb{N}^{*} \times \mathbb{N}^{*}}\right)$ is called a $\mathbb{G}$-mesh of $(X, \mathcal{M}, \mu)$. For abbreviation, we also say that $(X, \mathcal{M}, \mu)$ is $\mathbb{G}$-meshable with respect to $\left\{\mathbb{U}_{k}\right\}_{k \in \mathbb{N}^{*}}$.

The interest of Definition 2.1 comes from the following subadditive lemma (see Lemma 2.3) which is a key result for establishing subadditive ergodic theorems à la Akcoglu-Krengel (see $\$ 2.2$ ).

From now on, we assume that the measure space $(X, \mathcal{M}, \mu)$ is $\mathbb{G}$-meshable with a

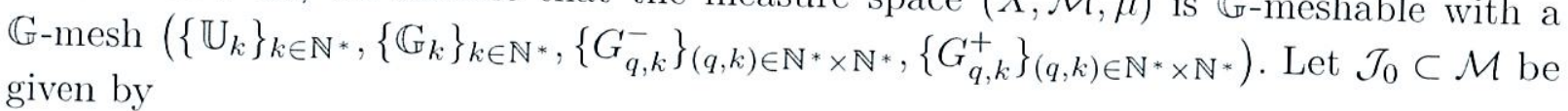

$$
\mathcal{J}_{0}:=\left\{\bigcup_{g \in H} \mathbb{U}_{k} g: k \in \mathbb{N}^{*}, H \in \mathcal{D}\left(\mathbb{U}_{k}\right) \text { and }|H|<\infty\right\}
$$


and let $\mathcal{J}$ be the $\mathbb{G}$-ring ${ }^{2}$ generated by $\mathcal{J}_{0}$. Let us recall the definition of a subadditive and $\mathbb{G}$-invariant set function.

Definition 2.2. Let $\mathcal{B}$ be a $\mathbb{G}$-ring and let $\mathcal{S}: \mathcal{B} \rightarrow \mathbb{R}$ be a set function.

(a) The set function $\mathcal{S}$ is said to be subadditive if

$$
\mathcal{S}\left(J \cup J^{\prime}\right) \leq \mathcal{S}(J)+\mathcal{S}\left(J^{\prime}\right)
$$

for all $J, J^{\prime} \in \mathcal{B}$ such that $A \cap B=\emptyset$.

(b) The set function $\mathcal{S}$ is said to be $\mathbb{G}$-invariant if

$$
\mathcal{S}(J g)=\mathcal{S}(J)
$$

for all $J \in \mathcal{B}$ and all $g \in \mathbb{G}$.

Lemma 2.3. Let $\mathcal{S}: \mathcal{J} \rightarrow \mathbb{R}$ be a subadditive and $\mathbb{G}$-invariant set function with the following boundedness condition: there exists $c>0$ such that

$$
|\mathcal{S}(A)| \leq c \mu(A)
$$

for all $A \in \mathcal{J}$. Then

$$
\lim _{q \rightarrow \infty} \frac{\mathcal{S}\left(\mathbb{U}_{q}\right)}{\mu\left(\mathbb{U}_{q}\right)}=\inf _{k \in \mathbb{N}^{*}} \frac{\mathcal{S}\left(\mathbb{U}_{k}\right)}{\mu\left(\mathbb{U}_{k}\right)}
$$

Proof of Lemma 2.3. First of all, it is clear that $\frac{\mathcal{S}\left(\mathbb{U}_{q}\right)}{\mu\left(\mathbb{U}_{q}\right)} \geq \inf _{k \in \mathbb{N}^{*}} \frac{\mathcal{S}\left(\mathbb{U}_{k}\right)}{\mu\left(\mathbb{U}_{k}\right)}$ for all $q \in \mathbb{N}^{*}$,
and so

$$
\varliminf_{q \rightarrow \infty} \frac{\mathcal{S}\left(\mathbb{U}_{q}\right)}{\mu\left(\mathbb{U}_{q}\right)} \geq \inf _{k \in \mathbb{N}^{*}} \frac{\mathcal{S}\left(\mathbb{U}_{k}\right)}{\mu\left(\mathbb{U}_{k}\right)} .
$$

On the other hand, fix any $k \in \mathbb{N}^{*}$ and any $q \in \mathbb{N}^{*}$ and set:

$$
\begin{aligned}
& J_{q, k}^{-}:=\underset{g \in G_{q, k}^{-}}{\cup} \mathbb{U}_{k} g ; \\
& J_{q, k}^{+}:=\underset{g \in G_{q, k}^{+}}{\cup} \mathbb{U}_{k} g .
\end{aligned}
$$

By the left inclusion in (2.1) we have $J_{q, k}^{-} \subset \mathbb{U}_{q}$ and so $\mathbb{U}_{q}=J_{q, k}^{-} \cup\left(\mathbb{U}_{q} \backslash J_{q, k}^{-}\right)$. Hence

$$
\mathcal{S}\left(\mathbb{U}_{q}\right) \leq \mathcal{S}\left(J_{q, k}^{-}\right)+\mathcal{S}\left(\mathbb{U}_{q} \backslash J_{q, k}^{-}\right)
$$

\footnotetext{
${ }^{2}$ We say that $\mathcal{J}$ is a $\mathbb{G}$-ring if $\mathcal{J}$ is a ring which is right stable by $\mathbb{G}$, i.e. $J g \in \mathcal{J}$ for all $J \in \mathcal{J}$ and all
$g \in \mathbb{G}$.
} 
because $\mathcal{S}$ is subadditive, and consequently

$$
\frac{\mathcal{S}\left(\mathbb{U}_{q}\right)}{\mu\left(\mathbb{U}_{q}\right)} \leq \frac{\mathcal{S}\left(J_{q, k}^{-}\right)}{\mu\left(J_{q, k}^{-}\right)} \frac{\mu\left(J_{q, k}^{-}\right)}{\mu\left(\mathbb{U}_{q}\right)}+\frac{\mathcal{S}\left(\mathbb{U}_{q} \backslash J_{q, k}^{-}\right)}{\mu\left(\mathbb{U}_{q}\right)}
$$

Using again the subadditivity of $\mathcal{S}$ and its $\mathbb{G}$-invariance (resp. the $\mathbb{G}$-invariance of $\mu$ ) we have

$$
\begin{aligned}
& \mathcal{S}\left(J_{q, k}^{-}\right) \leq\left|G_{q, k}^{-}\right| \mathcal{S}\left(\mathbb{U}_{k}\right) \\
& \left(\operatorname{resp.} \mu\left(J_{q, k}^{-}\right)=\left|G_{q, k}^{-}\right| \mu\left(\mathbb{U}_{k}\right)\right) .
\end{aligned}
$$

Moreover, $\mathbb{U}_{q} \subset J_{q, k}^{+}$by the right inclusion in (2.1), which implies that $\mathbb{U}_{q} \backslash J_{q, k}^{-} \subset$ $J_{q, k}^{+} \backslash J_{q, k}^{-}$and so

$$
\mathcal{S}\left(\mathbb{U}_{q} \backslash J_{q, k}^{-}\right) \leq c \mu\left(J_{q, k}^{+} \backslash J_{q, k}^{-}\right)
$$

with $c>0$ given by (2.3). It follows that

$$
\begin{aligned}
\frac{\mathcal{S}\left(\mathbb{U}_{q}\right)}{\mu\left(\mathbb{U}_{q}\right)} & \leq \frac{\mathcal{S}\left(\mathbb{U}_{k}\right)}{\mu\left(\mathbb{U}_{k}\right)} \frac{\mu\left(J_{q, k}^{-}\right)}{\mu\left(\mathbb{U}_{q}\right)}+\frac{c \mu\left(J_{q, k}^{+} \backslash J_{q, k}^{-}\right)}{\mu\left(\mathbb{U}_{q}\right)} \\
& \leq \frac{\mathcal{S}\left(\mathbb{U}_{k}\right)}{\mu\left(\mathbb{U}_{k}\right)}+\frac{c \mu\left(J_{q, k}^{+} \backslash J_{q, k}^{-}\right)}{\mu\left(\mathbb{U}_{q}\right)}
\end{aligned}
$$

because $\mu\left(J_{q, k}^{-}\right) \leq \mu\left(\mathbb{U}_{q}\right)$ since $J_{q, k}^{-} \subset \mathbb{U}_{q}$. Letting $q \rightarrow \infty$ and using $(2.2)$, and then passing to the infimum on $k$, we obtain

$$
\varlimsup_{n \rightarrow \infty} \frac{\mathcal{S}\left(\mathbb{U}_{q}\right)}{\mu\left(\mathbb{U}_{q}\right)} \leq \inf _{k \in \mathbb{N} *} \frac{\mathcal{S}\left(\mathbb{U}_{k}\right)}{\mu\left(\mathbb{U}_{k}\right)}
$$

and (2.4) follows by combining (2.5) with (2.6).

\subsection{Subadditive theorems à la Akcoglu-Krengel}

From now on, let $\left\{J_{n}\right\}_{n \in \mathbb{N}^{*}} \subset \mathcal{J}_{0}$. Then, for each $n \in \mathbb{N}^{*}$, one has

$$
J_{n}=\underset{g \in H_{n}}{\cup} \mathbb{U}_{q_{n}} g \text { with } H_{n} \in \mathcal{D}\left(\mathbb{U}_{q_{n}}\right)
$$




\subsubsection{The deterministic case}

We begin with the following definition.

Definition 2.4. We say that $\left\{J_{n}\right\}_{n \in \mathbb{N} *}$ is a $\mathbb{G}$-regular sequence if $q_{n} \rightarrow \infty$ and if for each $(n, k) \in \mathbb{N}^{*} \times \mathbb{N}^{*}$ there exist $\bar{q}_{n, k} \in \mathbb{N}^{*}, \bar{g}_{n, k} \in \mathbb{G}$ and $F_{n, k} \in \mathcal{P}_{\mathrm{f}}\left(\mathbb{G}_{k}\right)$ such that:

$$
\begin{aligned}
& G_{q_{n}, k}^{+} H_{n} \subset F_{n, k} ; \\
& \mathbb{U}_{\bar{q}_{n, k}} \bar{g}_{n, k}=\underset{r \in G_{q_{n}, k}^{+} H_{n}}{\cup} \mathbb{U}_{k} r ; \\
& \varlimsup_{n \rightarrow \infty} \frac{\left|F_{n, k}\right|}{\left|G_{q_{n}, k}^{+} H_{n}\right|}=1 .
\end{aligned}
$$

The following result generalizes Lemma 2.3.

Theorem 2.5. Let $\mathcal{S}: \mathcal{J} \rightarrow \mathbb{R}$ be a subadditive and $\mathbb{G}$-invariant set function satisfying (2.3). If $\left\{J_{n}\right\}_{n \in \mathbb{N} *}$ is a $\mathbb{G}$-regular sequence, then

$$
\lim _{n \rightarrow \infty} \frac{\mathcal{S}\left(J_{n}\right)}{\mu\left(J_{n}\right)}=\inf _{k \in \mathbb{N}^{*}} \frac{\mathcal{S}\left(\mathbb{U}_{k}\right)}{\mu\left(\mathbb{U}_{k}\right)} .
$$

Proof of Theorem 2.5. The proof is divided into four steps.

Step 1: using meshability. Using (2.1) we see that for each $(n, k) \in \mathbb{N}^{*} \times \mathbb{N}^{*}$, one has

$$
\underset{r \in G_{q_{n}, k}^{-} H_{n}}{\cup} \mathbb{U}_{k} r=\bigcup_{h \in H_{n}}\left(\underset{g \in G_{q_{n}, k}^{-}}{\cup} \mathbb{U}_{k} g\right) h \subset J_{n} \subset \underset{h \in H_{n}}{\cup}\left(\underset{g \in G_{q_{n}, k}^{+}}{\cup} \mathbb{U}_{k} g\right) h=\underset{r \in G_{q_{n}, k}^{+} H_{n}}{\cup} \mathbb{U}_{k} r .
$$

Moreover, since $F_{n, k} \in \mathcal{P}_{\mathrm{f}}\left(\mathbb{G}_{k}\right)$ and $G_{q_{n}, k}^{-} \subset G_{q_{n}, k}^{+}$, by $(2.7)$ we have $G_{q_{n}, k}^{-} H_{n} \subset$ $G_{q_{n}, k}^{+} H_{n} \in \mathcal{P}_{\mathrm{f}}\left(\mathbb{G}_{k}\right)$, and so $\left\{\mathbb{U}_{k} r\right\}_{r \in G_{q_{n}, k}^{-} H_{n}}$ and $\left\{\mathbb{U}_{k} r\right\}_{r \in G_{q_{n}, k}^{+} H_{n}}$ are disjoints. As $\mu$ is $\mathbb{G}$-invariant, it follows that

$$
\begin{aligned}
\frac{\mu\left(\cup_{r \in G_{q_{n}, k}^{+} H_{n}} \mathbb{U}_{k} r \backslash \cup_{r \in G_{q_{n}, k}^{-} H_{n}} \mathbb{U}_{k} r\right)}{\mu\left(J_{n}\right)} & =\left|G_{q_{n}, k}^{+} H_{n} \backslash G_{q_{n}, k}^{-} H_{n}\right| \mu\left(\mathbb{U}_{k}\right) \\
& =\frac{\left|H_{n}\right|\left|G_{q_{n}, k}^{+} \backslash G_{q_{n}, k}^{-}\right| \mu\left(\mathbb{U}_{k}\right)}{\left|H_{n}\right| \mu\left(\mathbb{U}_{q_{n}}\right)} \\
& =\left|G_{q_{n}, k}^{+} \backslash G_{q_{n}, k}^{-}\right| \frac{\mu\left(\mathbb{U}_{k}\right)}{\mu\left(\mathbb{U}_{q_{n}}\right)} .
\end{aligned}
$$

As $q_{n} \rightarrow \infty$, by using $(2.2)$ we deduce that

$$
\lim _{n \rightarrow \infty} \frac{\mu\left(\cup_{r \in G_{q_{n}, k}^{+} H_{n}} \mathbb{U}_{k} r \backslash \cup_{r \in G_{q_{n}, k}^{-} H_{n}} \mathbb{U}_{k} r\right)}{\mu\left(J_{n}\right)}=0 .
$$


Step 2: establishing lower bound and upper bound. Fix any $n \in \mathbb{N}^{*}$ and any $k \in \mathbb{N}^{*}$ and set:

$$
\begin{aligned}
& J_{n, k}^{-}:=\underset{r \in R_{n, k}^{-}}{\cup} \mathbb{U}_{k} r \\
& J_{n, k}^{+}:=\underset{g \in R_{n, k}^{+}}{\cup} \mathbb{U}_{k} r
\end{aligned}
$$

where $R_{n, k}^{-}:=G_{q_{n}, k}^{-} H_{n}$ and $R_{n, k_{j}}^{+}:=G_{q_{n}, k}^{+} H_{n}$.

Substep 2-1: lower bound. By the right inclusion in (2.11) we have $J_{n} \subset J_{n, k}^{+}$and so $J_{n, k}^{+}=J_{n} \cup\left(J_{n, k}^{+} \backslash J_{n}\right)$. Hence

$$
\mathcal{S}\left(J_{n, k}^{+}\right) \leq \mathcal{S}\left(J_{n}\right)+\mathcal{S}\left(J_{n, k}^{+} \backslash J_{n}\right)
$$

and consequently

$$
\frac{\mathcal{S}\left(J_{n, k}^{+}\right)}{\mu\left(J_{n, k}^{+}\right)} \leq \frac{\mathcal{S}\left(J_{n}\right)}{\mu\left(J_{n}\right)}+\frac{\mathcal{S}\left(J_{n, k}^{+} \backslash J_{n}\right)}{\mu\left(J_{n}\right)} .
$$

As $J_{n, k}^{-} \subset J_{n}$ by the left inclusion in (2.11), we see that $J_{n, k}^{+} \backslash J_{n} \subset J_{n, k}^{+} \backslash J_{n, k}^{-}$and so

$$
\mathcal{S}\left(J_{n, k}^{+} \backslash J_{n}\right) \leq c \mu\left(J_{n, k}^{+} \backslash J_{n, k}^{-}\right)
$$

with $c>0$ given by (2.3). It follows that

$$
\frac{\mathcal{S}\left(J_{n, k}^{+}\right)}{\mu\left(J_{n, k}^{+}\right)} \leq \frac{\mathcal{S}\left(J_{n}\right)}{\mu\left(J_{n}\right)}+\frac{c \mu\left(J_{n, k}^{+} \backslash J_{n, k}^{-}\right)}{\mu\left(J_{n}\right)} .
$$

Letting $n \rightarrow \infty$ and using (2.12) we obtain

$$
\underline{l}_{k}:=\underline{\lim }_{n \rightarrow \infty} \frac{\mathcal{S}\left(J_{n, k}^{+}\right)}{\mu\left(J_{n, k}^{+}\right)} \leq \underline{\lim _{n \rightarrow \infty}} \frac{\mathcal{S}\left(J_{n}\right)}{\mu\left(J_{n}\right)}=: \underline{l} .
$$

Substep 2-2: upper bound. By the left inclusion in (2.11) we have $J_{n, k}^{-} \subset J_{n}$ and so $J_{n}=J_{n, k}^{-} \cup\left(J_{n} \backslash J_{n, k}^{-}\right)$. Hence

$$
\mathcal{S}\left(J_{n}\right) \leq \mathcal{S}\left(J_{n, k}^{-}\right)+\mathcal{S}\left(J_{n} \backslash J_{n, k}^{-}\right),
$$

and consequently 


$$
\frac{\mathcal{S}\left(J_{n}\right)}{\mu\left(J_{n}\right)} \leq \frac{\mathcal{S}\left(J_{n, k}^{-}\right)}{\mu\left(J_{n, k}^{-}\right)} \frac{\mu\left(J_{n, k}^{-}\right)}{\mu\left(J_{n}\right)}+\frac{\mathcal{S}\left(J_{n} \backslash J_{n, k}^{-}\right)}{\mu\left(J_{n}\right)} .
$$

As $J_{n} \subset J_{n, k}^{+}$by the right inclusion in (2.11), we see that $J_{n} \backslash J_{n, k}^{-} \subset J_{n, k}^{+} \backslash J_{n, k}^{-}$and so

$$
\mathcal{S}\left(J_{n} \backslash J_{n, k}^{-}\right) \leq c \mu\left(J_{n, k}^{+} \backslash J_{n, k}^{-}\right)
$$

with $c>0$ given by (2.3). It follows that

$$
\begin{aligned}
\frac{\mathcal{S}\left(J_{n}\right)}{\mu\left(J_{n}\right)} & \leq \frac{\mathcal{S}\left(J_{n, k}^{-}\right)}{\mu\left(J_{n, k}^{-}\right)} \frac{\mu\left(J_{n, k}^{-}\right)}{\mu\left(J_{n}\right)}+\frac{c \mu\left(J_{n, k}^{+} \backslash J_{n, k}^{-}\right)}{\mu\left(J_{n}\right)} \\
& \leq \frac{\mathcal{S}\left(J_{n, k}^{-}\right)}{\mu\left(J_{n, k}^{-}\right)}+\frac{c \mu\left(J_{n, k}^{+} \backslash J_{n, k}^{-}\right)}{\mu\left(J_{n}\right)}
\end{aligned}
$$

because $\mu\left(J_{n, k}^{-}\right) \leq \mu\left(J_{n}\right)$ since $J_{n, k}^{-} \subset J_{n}$. Letting $n \rightarrow \infty$ and using (2.12) we obtain

$$
\bar{l}:=\varlimsup_{n \rightarrow \infty} \frac{\mathcal{S}\left(J_{n}\right)}{\mu\left(J_{n}\right)} \leq \varlimsup_{n \rightarrow \infty} \frac{\mathcal{S}\left(J_{n, k}^{-}\right)}{\mu\left(J_{n, k}^{-}\right)}=: \bar{l}_{k} .
$$

Step 3: we prove that $\underline{l}=\bar{l}$. It is sufficient to prove that for each $\varepsilon>0$, one has

$$
\bar{l}-\underline{l}<\varepsilon .
$$

Fix $\varepsilon>0$. From (2.13) and (2.14) we see that $\bar{l}-\underline{l} \leq \bar{l}_{k}-\underline{l}_{k}$. So, to prove (2.15) it suffices to show that there exists $k \in \mathbb{N}^{*}$ such that

$$
\bar{l}_{k}-\underline{l}_{k}<\varepsilon .
$$

Let $\mathcal{S}_{k}: \mathcal{P}_{\mathrm{f}}\left(\mathbb{G}_{k}\right) \rightarrow \mathbb{R}$ be defined by

$$
\mathcal{S}_{k}(E):=\frac{1}{\mu\left(\mathbb{U}_{k}\right)}\left[\mathcal{S}\left(\underset{r \in E}{\cup} \mathbb{U}_{k} r\right)-|E| \mathcal{S}\left(\mathbb{U}_{k}\right)\right] .
$$

As $\mathcal{S}$ is subadditive, we can assert that $\mathcal{S}_{k}$ is negative, i.e.,

$$
\mathcal{S}_{k}(E)=\frac{1}{\mu\left(\mathbb{U}_{k}\right)}\left[\mathcal{S}\left(\underset{r \in E}{\cup} \mathbb{U}_{k} r\right)-|E| \mathcal{S}\left(\mathbb{U}_{k}\right)\right] \leq 0
$$

for all $E \in \mathcal{P}_{\mathrm{f}}\left(\mathbb{G}_{k}\right)$. Moreover, it is easily seen that $\mathcal{S}_{k}$ is decreasing, i.e., for all $E, F \in$ $\mathcal{P}_{\mathrm{f}}\left(\mathbb{G}_{k}\right)$, if $E \subset F$ then $\mathcal{S}_{k}(E) \geq \mathcal{S}_{k}(F)$. Consider $\bar{q}_{n, k} \in \mathbb{N}^{*}, \bar{g}_{n, k} \in \mathbb{G}$ and $F_{n, k} \in \mathcal{P}_{\mathrm{f}}\left(\mathbb{G}_{k}\right)$ given by Definition 2.4. From (2.8) it follows that 


$$
\begin{aligned}
\mathcal{S}_{k}\left(R_{n, k}^{+}\right) \geq \mathcal{S}_{k}\left(F_{n, k}\right) & =\frac{1}{\mu\left(\mathbb{U}_{k}\right)}\left[\mathcal{S}\left(\underset{r \in F_{n, k}}{\cup} \mathbb{U}_{k} r\right)-\left|F_{n, k}\right| \mathcal{S}\left(\mathbb{U}_{k}\right)\right] \\
& =\frac{1}{\mu\left(\mathbb{U}_{k}\right)}\left[\mathcal{S}\left(\mathbb{U}_{\bar{q}_{n, k}} \bar{g}_{n, k}\right)-\left|F_{n, k}\right| \mathcal{S}\left(\mathbb{U}_{k}\right)\right] .
\end{aligned}
$$

Hence, since $\frac{1}{\left|R_{n, k}^{+}\right|} \geq \frac{1}{\left|F_{n, k}\right|}$ and $\mathcal{S}$ and $\mu$ are $\mathbb{G}$-invariant, we get

$$
\begin{aligned}
\frac{\mathcal{S}_{k}\left(R_{n, k}^{+}\right)}{\left|R_{n, k}^{+}\right|} & \geq \frac{1}{\left|R_{n, k}^{+}\right| \mu\left(\mathbb{U}_{k}\right)}\left[\mathcal{S}\left(\mathbb{U}_{\bar{q}_{n, k}} \bar{g}_{n, k}\right)-\left|F_{n, k}\right| \mathcal{S}\left(\mathbb{U}_{k}\right)\right] \\
& \geq \frac{\mathcal{S}\left(\mathbb{U}_{\bar{q}_{n, k}} \bar{g}_{n, k}\right)}{\left|F_{n, k}\right| \mu\left(\mathbb{U}_{k}\right)}-\frac{\left|F_{n, k}\right|}{\left|R_{n, k}^{+}\right|} \frac{\mathcal{S}\left(\mathbb{U}_{k}\right)}{\mu\left(\mathbb{U}_{k}\right)} \\
& =\frac{\mathcal{S}\left(\mathbb{U}_{\bar{q}_{n, k}} \bar{g}_{n, k}\right)}{\mu\left(\mathbb{U}_{\bar{q}_{n, k}} \bar{g}_{n, k}\right)}-\frac{\left|F_{n, k}\right|}{\left|R_{n, k}^{+}\right|} \frac{\mathcal{S}\left(\mathbb{U}_{k}\right)}{\mu\left(\mathbb{U}_{k}\right)} \\
& =\frac{\mathcal{S}\left(\mathbb{U}_{\bar{q}_{n, k}}\right)}{\mu\left(\mathbb{U}_{\bar{q}_{n, k}}\right)}-\frac{\left|F_{n, k}\right|}{\left|R_{n, k}^{+}\right|} \frac{\mathcal{S}\left(\mathbb{U}_{k}\right)}{\mu\left(\mathbb{U}_{k}\right)} \\
& \geq \inf _{q \in \mathbb{N}^{*}} \frac{\mathcal{S}\left(\mathbb{U}_{q}\right)}{\mu\left(\mathbb{U}_{q}\right)}-\frac{\left|F_{n, k}\right|}{\left|R_{n, k}^{+}\right|} \frac{\mathcal{S}\left(\mathbb{U}_{k}\right)}{\mu\left(\mathbb{U}_{k}\right)}
\end{aligned}
$$

Letting $n \rightarrow \infty$ and taking (2.9) into account, we deduce that

$$
\varliminf_{n \rightarrow \infty} \frac{\mathcal{S}_{k}\left(R_{n, k}^{+}\right)}{\left|R_{n, k}^{+}\right|} \geq \inf _{q \in \mathbb{N}^{*}} \frac{\mathcal{S}\left(\mathbb{U}_{q}\right)}{\mu\left(\mathbb{U}_{q}\right)}-\frac{\mathcal{S}\left(\mathbb{U}_{k}\right)}{\mu\left(\mathbb{U}_{k}\right)}
$$

By Lemma 2.3 we can assert that there exists $k_{\varepsilon} \in \mathbb{N}^{*}$ such that for each $k \geq k_{\varepsilon}$, one has

$$
\frac{\mathcal{S}\left(\mathbb{U}_{k}\right)}{\mu\left(\mathbb{U}_{k}\right)}-\inf _{q \in \mathbb{N}^{*}} \frac{\mathcal{S}\left(\mathbb{U}_{q}\right)}{\mu\left(\mathbb{U}_{q}\right)}<\varepsilon .
$$

Combining (2.19) with (2.20) we conclude that

$$
\varliminf_{n \rightarrow \infty} \frac{\mathcal{S}_{k}\left(R_{n, k}^{+}\right)}{\left|R_{n, k}^{+}\right|}>-\varepsilon
$$

for all $k \geq k_{\varepsilon}$. On the other hand, by using (2.17) with $E=R_{n, k}^{+}$and (2.18) with $E=R_{n, k}^{-}$we get:

$$
\frac{\mathcal{S}\left(J_{n, k}^{+}\right)}{\mu\left(J_{n, k}^{+}\right)}-\frac{\mathcal{S}\left(\mathbb{U}_{k}\right)}{\mu\left(\mathbb{U}_{k}\right)}=\frac{\mathcal{S}_{k}\left(R_{n, k}^{+}\right)}{\left|R_{n, k}^{+}\right|} ;
$$




$$
\frac{\mathcal{S}\left(J_{n, k}^{-}\right)}{\mu\left(J_{n, k}^{-}\right)}-\frac{\mathcal{S}\left(\mathbb{U}_{k}\right)}{\mu\left(\mathbb{U}_{k}\right)} \leq 0 .
$$

Letting $n \rightarrow \infty$ in (2.22) and (2.23) and taking (2.21) into account, we deduce that:

$$
\begin{aligned}
& \underline{l}_{k}-\frac{\mathcal{S}\left(\mathbb{U}_{k}\right)}{\mu\left(\mathbb{U}_{k}\right)}>-\varepsilon \text { for all } k \geq k_{\varepsilon} ; \\
& \bar{l}_{k}-\frac{\mathcal{S}\left(\mathbb{U}_{k}\right)}{\mu\left(\mathbb{U}_{k}\right)} \leq 0 \text { for all } k \in \mathbb{N}^{*},
\end{aligned}
$$

and (2.16) follows with $j=j_{\varepsilon}$. We set $l:=\underline{l}=\bar{l}$ and $\gamma:=\inf _{q \in \mathbb{N} *} \frac{\mathcal{S}\left(\mathbb{U}_{q}\right)}{\mu\left(\mathbb{U}_{q}\right)}$.

Step 4: we prove that $l=\gamma$. Combining (2.14) with (2.25) we see that $l \leq \frac{\mathcal{S}\left(\mathbb{U}_{k}\right)}{\mu\left(\mathbb{U}_{k}\right)}$ for all $k \in \mathbb{N}^{*}$, and so $l \leq \gamma$ by letting $k \rightarrow \infty$ and using Lemma 2.3. On the other hand, combining (2.13) with (2.24) we see that $l>-\varepsilon+\frac{\mathcal{S}\left(\mathbb{U}_{k}\right)}{\mu\left(\mathbb{U}_{k}\right)}$ for all $k \geq k_{\varepsilon}$. Letting $k \rightarrow \infty$ and using Lemma 2.3 we deduce that $l \geq-\varepsilon+\gamma$ for all $\varepsilon>0$, and so $l \geq \gamma$ by letting $\varepsilon \rightarrow 0$.

\subsubsection{The stochastic case}

In what follows, for each $h \in \mathbb{G}$ and each $E, F \subset \mathbb{G}$, we adopt the following notation:

- $E \Delta F:=(E \backslash F) \cup(F \backslash E)$;

- $E F:=\{g f:(g, f) \in E \times F\}$;

- $E^{-1} F:=\left\{g^{-1} f:(g, f) \in E \times F\right\}$;

- $h E:=\{h g: g \in E\}$.

We begin with the definition of amenability. (For more details about the theory of amenability, we refer to $[17,36,37,41,1,16]$ and the references therein, see also $[27, \S 6.4]$.)

Definition 2.6. Let $H \subset \mathbb{G}$ be a subgroup. We say that $H$ is amenable if for each $E \in$ $\mathcal{P}_{\mathrm{f}}(H)$ and each $\delta>0$ there exists $F \in \mathcal{P}_{\mathrm{f}}(H)$ such that $|F \Delta E F| \leq \delta|F|$.

The property of Følner-Tempelman stated in the definition below is needed to use both Lindenstrauss's ergodic theorem (see Theorem 2.16) which is valid for general amenable groups and a maximal inequality (see Lemma 2.17) which is valid for countable discrete amenable groups. (These two results will be used in the proof of Theorem 2.14.)

Definition 2.7. Let $H$ be an amenable subgroup of $\mathbb{G}$ and let $\left\{R_{n}\right\}_{n \in \mathbb{N}^{*}} \subset \mathcal{P}_{\mathrm{f}}(H)$. We say that $\left\{R_{n}\right\}_{n \in \mathbb{N}^{*}}$ is of Følner-Tempelman type with respect to $H$ if it satisfies the following two conditions: 
(a) Følner's condition: for every $g \in H$, one has

$$
\lim _{n \rightarrow \infty} \frac{\left|g R_{n} \Delta R_{n}\right|}{\left|R_{n}\right|}=0
$$

(b) Tempelman's condition: there exists $M>0$, which called the Templeman constant associated with $\left\{R_{n}\right\}_{n \in \mathbb{N}^{*}}$, such that for every $n \in \mathbb{N}^{*}$, one has

$$
\left|\bigcup_{i=1}^{n} R_{i}^{-1} R_{n}\right| \leq M\left|R_{n}\right|
$$

From now on, the mesh $\left(\left\{\mathbb{U}_{k}\right\}_{k \in \mathbb{N}^{*}},\left\{\mathbb{G}_{k}\right\}_{k \in \mathbb{N}^{*}},\left\{G_{q, k}^{-}\right\}_{(q, k) \in \mathbb{N}^{*} \times \mathbb{N}^{*}},\left\{G_{q, k}^{+}\right\}_{(q, k) \in \mathbb{N}^{*} \times \mathbb{N}^{*}}\right)$ is assumed to satisfy the following two additional conditions:

$$
\begin{aligned}
& \mathbb{G}_{k} \text { is a countable discrete amenable subgroup of } \mathbb{G} \text { for all } k \in \mathbb{N}^{*} \\
& \cup_{k \in \mathbb{N}^{*}} \mathbb{G}_{k}=\mathbb{G} \text {. }
\end{aligned}
$$

Definition 2.8. When (2.26) and (2.27) are satisfied, we say that $(X, \mathcal{M}, \mu)$ is strongly $\mathbb{G}$-meshable.

We also need to strengthen Definition 2.4 as follows.

Definition 2.9. We say that $\left\{J_{n}\right\}_{n \in \mathbb{N}^{*}}$ is a strongly $\mathbb{G}$-regular sequence if it is $\mathbb{G}$-regular sequence with the additional assumption that for all $k \in \mathbb{N}^{*},\left\{G_{q_{n}, k}^{-} H_{n}\right\}_{n \in \mathbb{N}^{*}}$ and $\left\{G_{q_{n}, k}^{+} H_{n}\right\}_{n \in \mathbb{N}^{*}}$ are of Følner-Tempelman type with respect to $\mathbb{G}_{k}$.

Let $(\Omega, \mathcal{F}, \mathbb{P})$ be a probability space and let $\left\{\tau_{g}: \Omega \rightarrow \Omega\right\}_{g \in \mathbb{G}}$ be satisfying the following three properties:

- $\tau_{g}$ is $\mathcal{F}$-measurable for all $g \in \mathbb{G}$;

- $\tau_{g} \circ \tau_{f}=\tau_{g \circ f}$ and $\tau_{g^{-1}}=\tau_{g}^{-1}$ for all $g, f \in \mathbb{G}$;

- $\mathbb{P}\left(\tau_{g}(A)\right)=\mathbb{P}(A)$ for all $A \in \mathcal{F}$ and all $g \in \mathbb{G}$.

Definition 2.10. Such a $\left\{\tau_{g}\right\}_{g \in \mathbb{G}}$ is said to be a group of $\mathbb{P}$-preserving transformation on $(\Omega, \mathcal{F}, \mathbb{P})$ and the quadruplet $\left(\Omega, \mathcal{F}, \mathbb{P},\left\{\tau_{g}\right\}_{g \in \mathbb{G}}\right)$ is called a measurable dynamical $\mathbb{G}$-system.

Remark 2.11. If $\left(\Omega, \mathcal{F}, \mathbb{P},\left\{\tau_{g}\right\}_{g \in \mathbb{G}}\right)$ is a measurable dynamical $\mathbb{G}$-system, then for each subgroup $H$ of $\mathbb{G},\left(\Omega, \mathcal{F}, \mathbb{P},\left\{\tau_{h}\right\}_{h \in H}\right)$ is a measurable dynamical $H$-system.

Let $\mathcal{I}:=\left\{A \in \mathcal{F}: \mathbb{P}\left(\tau_{g}(A) \Delta A\right)=0\right.$ for all $\left.g \in \mathbb{G}\right\}$ be the $\sigma$-algebra of invariant sets with respect to $\left(\Omega, \mathcal{F}, \mathbb{P},\left\{\tau_{g}\right\}_{g \in \mathbb{G}}\right.$ ). (For each subgroup $H$ of $\mathbb{G}$, we denote the $\sigma$-algebra of invariant sets with respect to $\left(\Omega, \mathcal{F}, \mathbb{P},\left\{\tau_{g}\right\}_{g \in H}\right)$ by $\mathcal{I}_{H}$.) 
Definition 2.12. When $\mathbb{P}(A) \in\{0,1\}$ for all $A \in \mathcal{I}$, the measurable dynamical $\mathbb{G}$-system $\left(\Omega, \mathcal{F}, \mathbb{P},\left\{\tau_{g}\right\}_{g \in \mathbb{G}}\right)$ is said to be ergodic.

In what follows, we assume that $\left(\Omega, \mathcal{F}, \mathbb{P},\left\{\tau_{g}\right\}_{g \in \mathbb{G}}\right)$ is a measurable dynamical $\mathbb{G}$ system. Let us recall the definition of a subadditive process.

Definition 2.13. Let $\mathcal{B}$ be a $\mathbb{G}$-ring. We say that $\mathcal{S}: \mathcal{B} \rightarrow L^{1}(\Omega, \mathcal{F}, \mathbb{P})$ is a subadditive process if $\mathcal{S}$ is subadditive in the sense of Definition 2.2(a) and $\mathbb{G}$-covariant, i.e.

$$
\mathcal{S}(J g)=\mathcal{S}(J) \circ \tau_{g}
$$

for all $J \in \mathcal{B}$ and all $g \in \mathbb{G}$. If in addition $\left(\Omega, \mathcal{F}, \mathbb{P},\left\{\tau_{g}\right\}_{g \in \mathbb{G}}\right)$ is ergodic, then $\mathcal{S}$ is called an ergodic subadditive process.

The following result is an extension of Akcoglu-Krengel's subadditive theorem to the setting of measure spaces with acting groups.

Theorem 2.14. Let $\mathcal{S}: \mathcal{J} \rightarrow L^{1}(\Omega, \mathcal{F}, \mathbb{P})$ be a subadditive process satisfying (2.3). If $\left\{J_{n}\right\}_{n \in \mathbb{N}^{*}}$ is a strongly $\mathbb{G}$-regular sequence, then

$$
\lim _{n \rightarrow \infty} \frac{\mathcal{S}\left(J_{n}\right)(\omega)}{\mu\left(J_{n}\right)}=\inf _{k \in \mathbb{N}^{*}} \frac{\mathbb{E}^{\mathcal{I}}\left[\mathcal{S}\left(\mathbb{U}_{k}\right)\right](\omega)}{\mu\left(\mathbb{U}_{k}\right)} \text { for } \mathbb{P} \text {-a.a. } \omega \in \Omega,
$$

where $\mathbb{E}^{\mathcal{I}}\left[\mathcal{S}\left(\mathbb{U}_{k}\right)\right]$ denotes the conditional expectation of $\mathcal{S}\left(\mathbb{U}_{k}\right)$ over $\mathcal{I}$ with respect to $\mathbb{P}$. If in addition $\left(\Omega, \mathcal{F}, \mathbb{P},\left\{\tau_{g}\right\}_{g \in \mathbb{G}}\right)$ is ergodic, then

$$
\lim _{n \rightarrow \infty} \frac{\mathcal{S}\left(J_{n}\right)(\omega)}{\mu\left(J_{n}\right)}=\inf _{k \in \mathbb{N}^{*}} \frac{\mathbb{E}\left[\mathcal{S}\left(\mathbb{U}_{k}\right)\right]}{\mu\left(\mathbb{U}_{k}\right)} \text { for } \mathbb{P}-\text { a.a. } \omega \in \Omega
$$

where $\mathbb{E}\left[\mathcal{S}\left(\mathbb{U}_{k}\right)\right]$ denotes the expectation of $\mathcal{S}\left(\mathbb{U}_{k}\right)$ with respect to $\mathbb{P}$.

Proof of Theorem 2.14. The proof is divided into four steps.

Step 1: establishing lower bound and upper bound. Fix any $k \in \mathbb{N}^{*}$ and any $n \in \mathbb{N}^{*}$ and set:

$$
\begin{aligned}
& J_{n, k}^{-}:=\bigcup_{r \in R_{n, k}^{-}} \mathbb{U}_{k} r ; \\
& J_{n, k}^{+}:=\bigcup_{r \in R_{n, k}^{+}} \mathbb{U}_{k} r,
\end{aligned}
$$

where $R_{n, k}^{-}:=G_{q_{n}, k}^{-} H_{n}$ and $R_{n, k_{j}}^{+}:=G_{q_{n}, k}^{+} H_{n}$. Arguing as in Step 1 of the proof of Theorem 2.5 we see that $(2.11)$ and $(2.12)$ hold, and using the same approximation argument as in Step 2 of the proof of Theorem 2.5, for each $\omega \in \Omega$, we get: 


$$
\begin{aligned}
& \underline{l}_{k}(\omega):=\varliminf_{n \rightarrow \infty} \frac{\mathcal{S}\left(J_{n, k}^{+}\right)(\omega)}{\mu\left(J_{n, k}^{+}\right)} \leq \underline{\lim _{n \rightarrow \infty} \frac{\mathcal{S}\left(J_{n}\right)(\omega)}{\mu\left(J_{n}\right)}=: \underline{l}(\omega) ;} \\
& \bar{l}(\omega):=\varlimsup_{n \rightarrow \infty} \frac{\mathcal{S}\left(J_{n}\right)(\omega)}{\mu\left(J_{n}\right)} \leq \varlimsup_{n \rightarrow \infty} \frac{\mathcal{S}\left(J_{n, k}^{-}\right)(\omega)}{\mu\left(J_{n, k}^{-}\right)}=: \bar{l}_{k}(\omega) .
\end{aligned}
$$

Remark 2.15. Arguing as in Step 2-1 of the proof of Theorem 2.5, we see that we also have

$$
\varlimsup_{n \rightarrow \infty} \frac{\mathcal{S}\left(J_{n, k}^{+}\right)(\omega)}{\mu\left(J_{n, k}^{+}\right)} \leq \bar{l}(\omega)
$$

for all $\omega \in \Omega$. (This will be used in Step 3.)

Step 2: we prove that $\underline{\boldsymbol{l}}=\overline{\boldsymbol{l}}$. It is sufficient to prove that for each $\alpha>0$, one has

$$
\mathbb{P}(\{\omega \in \Omega: \bar{l}(\omega)-\underline{l}(\omega)>\alpha\})=0
$$

Fix $\alpha>0$. From (2.28) and (2.29) we see that for each $k \in \mathbb{N}^{*}$, one has

$$
\{\omega \in \Omega: \bar{l}(\omega)-\underline{l}(\omega)>\alpha\} \subset\left\{\omega \in \Omega: \bar{l}_{k}(\omega)-\underline{l}_{k}(\omega)>\alpha\right\}=: W_{k, \alpha}
$$

So, to prove (2.31) it suffices to show that for each $\varepsilon>0$ there exists $k \in \mathbb{N}^{*}$ such that

$$
\mathbb{P}\left(W_{k, \alpha}\right) \leq \frac{M_{k}}{\alpha} \varepsilon
$$

where $M_{k}>0$ is the Tempelman constant associated with $\left\{R_{n, k}^{+}\right\}_{n \in \mathbb{N}^{*}}$. Fix $\varepsilon>0$.

Substep 2-1: constructing a decreasing negative subadditive process. Let $\mathcal{A}_{k}: \mathcal{P}_{\mathrm{f}}\left(\mathbb{G}_{k}\right) \rightarrow$ $L^{1}(\Omega, \mathcal{F}, \mathbb{P})$ be defined by

$$
\mathcal{A}_{k}(E):=\sum_{r \in E} \mathcal{S}\left(\mathbb{U}_{k}\right) \text { o } \tau_{r}
$$

and let $\mathcal{S}_{k}: \mathcal{P}_{\mathrm{f}}\left(\mathbb{G}_{k}\right) \rightarrow L^{1}(\Omega, \mathcal{F}, \mathbb{P})$ be defined by

$$
\mathcal{S}_{k}(E):=\frac{1}{\mu\left(\mathbb{U}_{k}\right)}\left[\mathcal{S}\left(\cup_{r \in E} \mathbb{U}_{k} r\right)-\mathcal{A}_{k}(E)\right]
$$


As $\mathcal{S}$ is subadditive and $\mathbb{G}$-covariant (and so $\mathbb{G}_{k^{-}}$-covariant) and $\mathcal{A}_{k}$ is additive and $\mathbb{G}_{k^{-}}$ covariant, we can assert that $\mathcal{S}_{k}$ is a subadditive process ${ }^{3}$ on $\mathcal{P}_{\mathrm{f}}\left(\mathbb{G}_{k}\right)$ which is negative, i.e.

$$
\mathcal{S}_{k}(E)(\omega)=\frac{1}{\mu\left(\mathbb{U}_{k}\right)}\left[\mathcal{S}\left(\cup_{r \in E} \mathbb{U}_{k} r\right)(\omega)-\mathcal{A}_{k}(E)(\omega)\right] \leq 0
$$

for all $E \in \mathcal{P}_{\mathrm{f}}\left(\mathbb{G}_{k}\right)$ and all $\omega \in \Omega$. Moreover, it is easily seen that $\mathcal{S}_{k}$ is decreasing, i.e. for all $E, F \in \mathcal{P}_{\mathrm{f}}\left(\mathbb{G}_{k}\right)$, if $E \subset F$ then $\mathcal{S}_{k}(E) \geq \mathcal{S}_{k}(F)$. Consider $\bar{q}_{n, k} \in \mathbb{N}^{*}, \bar{g}_{n, k} \in \mathbb{G}$ and $F_{n, k} \in \mathcal{P}_{\mathrm{f}}\left(\mathbb{G}_{k}\right)$ given by Definition 2.4. From (2.8) it follows that

$$
\begin{aligned}
\mathcal{S}_{k}\left(R_{n, k}^{+}\right) \geq \mathcal{S}_{k}\left(F_{n, k}\right) & =\frac{1}{\mu\left(\mathbb{U}_{k}\right)}\left[\mathcal{S}\left(\underset{r \in F_{n, k}}{\cup} \mathbb{U}_{k} r\right)-\mathcal{A}_{k}\left(F_{n, k}\right)\right] \\
& =\frac{1}{\mu\left(\mathbb{U}_{k}\right)}\left[\mathcal{S}\left(\mathbb{U}_{\bar{q}_{n, k}} \bar{g}_{n, k}\right)-\mathcal{A}_{k}\left(F_{n, k}\right)\right] .
\end{aligned}
$$

By using the $\mathbb{G}$-covariance of $\mathcal{S}$ we see that

$$
\begin{aligned}
\int_{\Omega} \mathcal{S}_{k}\left(R_{n, k}^{+}\right)(\omega) d \mathbb{P}(\omega) & \geq \frac{1}{\mu\left(\mathbb{U}_{k}\right)}\left[\int_{\Omega} \mathcal{S}\left(\mathbb{U}_{\bar{q}_{n, k}} \bar{g}_{n, k}\right)(\omega) d \mathbb{P}(\omega)-\int_{\Omega} \mathcal{A}_{k}\left(F_{n, k}\right)(\omega) d \mathbb{P}(\omega)\right] \\
& =\frac{1}{\mu\left(\mathbb{U}_{k}\right)}\left[\int_{\Omega} \mathcal{S}\left(\mathbb{U}_{\bar{q}_{n, k}}\right)(\omega) d \mathbb{P}(\omega)-\left|F_{n, k}\right| \mathbb{E}\left[\mathcal{S}\left(\mathbb{U}_{k}\right)\right]\right] \\
& =\frac{\mathbb{E}\left[\mathcal{S}\left(\mathbb{U}_{\bar{q}_{n, k}}\right)\right]}{\mu\left(\mathbb{U}_{k}\right)}-\left|F_{n, k}\right| \frac{\mathbb{E}\left[\mathcal{S}\left(\mathbb{U}_{k}\right)\right]}{\mu\left(\mathbb{U}_{k}\right)} .
\end{aligned}
$$

Consequently, since $\frac{1}{\left|R_{n, k}^{+}\right|} \geq \frac{1}{\left|F_{n, k}\right|}$ and $\mu$ is $\mathbb{G}$-invariant, we get

$$
\begin{aligned}
\frac{\mathbb{E}\left[\mathcal{S}_{k}\left(R_{n, k}^{+}\right)\right]}{\left|R_{n, k}^{+}\right|} & \geq \frac{\mathbb{E}\left[\mathcal{S}\left(\mathbb{U}_{\bar{q}_{n, k}}\right)\right]}{\mu\left(\mathbb{U}_{\bar{q}_{n, k}}\right)}-\frac{\left|F_{n, k}\right|}{\left|R_{n, k}^{+}\right|} \frac{\mathbb{E}\left[\mathcal{S}\left(\mathbb{U}_{k}\right)\right]}{\mu\left(\mathbb{U}_{k}\right)} \\
& \geq \inf _{q \in \mathbb{N}^{*}} \frac{\mathbb{E}\left[\mathcal{S}\left(\mathbb{U}_{q}\right)\right]}{\mu\left(\mathbb{U}_{q}\right)}-\frac{\left|F_{n, k}\right|}{\left|R_{n, k}^{+}\right|} \frac{\mathbb{E}\left[\mathcal{S}\left(\mathbb{U}_{k}\right)\right]}{\mu\left(\mathbb{U}_{k}\right)}
\end{aligned}
$$

Letting $n \rightarrow \infty$ and taking (2.9) into account, we deduce that

$$
\varliminf_{n \rightarrow \infty} \frac{\mathbb{E}\left[\mathcal{S}_{k}\left(R_{n, k}^{+}\right)\right]}{\left|R_{n, k}^{+}\right|} \geq \inf _{q \in \mathbb{N}^{*}} \frac{\mathbb{E}\left[\mathcal{S}\left(\mathbb{U}_{q}\right)\right]}{\mu\left(\mathbb{U}_{q}\right)}-\frac{\mathbb{E}\left[\mathcal{S}\left(\mathbb{U}_{k}\right)\right]}{\mu\left(\mathbb{U}_{k}\right)} .
$$

\footnotetext{
${ }^{3}$ The set function $\mathcal{S}_{k}: \mathcal{P}_{\mathrm{f}}\left(\mathbb{G}_{k}\right) \rightarrow L^{1}(\Omega, \mathcal{F}, \mathbb{P})$ is said to be a subadditive process on $\mathcal{P}_{\mathrm{f}}\left(\mathbb{G}_{k}\right)$ if $\mathcal{S}_{k}$ is subadditive, i.e. $\mathcal{S}_{k}(E \cup F) \leq \mathcal{S}_{k}(E)+\mathcal{S}_{k}(F)$ for all $E, F \in \mathcal{P}_{\mathrm{f}}\left(\mathbb{G}_{k}\right)$ such that $E \cap F=\emptyset$, and $\mathbb{G}_{k}$-covariant, i.e. $\mathcal{S}_{k}(E r)=\mathcal{S}_{k}(E)$ o $\tau_{r}$ for all $E \in \mathcal{P}_{\mathrm{f}}\left(\mathbb{G}_{k}\right)$ and all $r \in \mathbb{G}_{k}$.
} 
As $\mathcal{S}$ is subadditive and $\mathbb{G}$-covariant, we see that the set function $\mathbb{E}[\mathcal{S}(\cdot)]$ is subadditive and $\mathbb{G}$-invariant. From Lemma 2.3 it follows that there exists $k_{\varepsilon} \in \mathbb{N}^{*}$ such that for each $k \geq k_{\varepsilon}$, one has

$$
\frac{\mathbb{E}\left[\mathcal{S}\left(\mathbb{U}_{k}\right)\right]}{\mu\left(\mathbb{U}_{k}\right)}-\inf _{q \in \mathbb{N}^{*}} \frac{\mathbb{E}\left[\mathcal{S}\left(\mathbb{U}_{q}\right)\right]}{\mu\left(\mathbb{U}_{q}\right)}<\varepsilon
$$

Combining (2.36) with (2.37) we conclude that

$$
\varlimsup_{n \rightarrow \infty} \frac{\mathbb{E}\left[\mathcal{S}_{k}\left(R_{n, k}^{+}\right)\right]}{\left|R_{n, k}^{+}\right|}>-\varepsilon
$$

for all $k \geq k_{\varepsilon}$.

Substep 2-2: using Lindenstrauss's ergodic theorem. We need the following pointwise additive ergodic theorem ${ }^{4}$ due to Lindenstrauss (see [29, Theorem 1.2] and also [12, Theorem 2.1]).

Theorem 2.16. Let $\Theta \in L^{1}(\Omega, \mathcal{F}, \mathbb{P})$ and let $\left\{R_{n}\right\}_{n \in \mathbb{N}^{*}} \subset \mathcal{P}_{\mathrm{f}}\left(\mathbb{G}_{k}\right)$. If $\left\{R_{n}\right\}_{n \in \mathbb{N}^{*}}$ is of Følner-Tempelman type with respect to $\mathbb{G}_{k}$ then

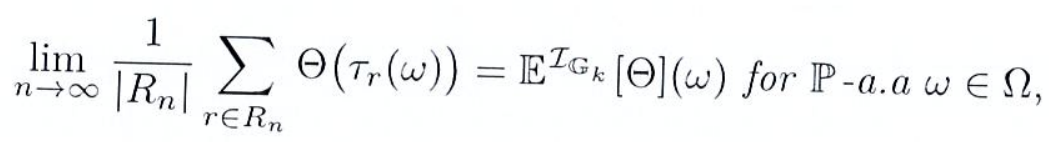

where $\mathcal{I}_{\mathbb{G}_{k}}$ is the $\sigma$-algebra of invariant sets with respect to $\left(\Omega, \mathcal{F}, \mathbb{P},\left\{\tau_{r}\right\}_{r \in \mathbb{G}_{k}}\right)$ and $\mathbb{E}^{\mathcal{I}_{\mathbb{G}_{k}}}[\Theta]$ denotes the conditional expectation over $\mathcal{I}_{\mathbb{G}_{k}}$ with respect to $\mathbb{P}$.

As $\left\{R_{n, k}^{-}\right\}_{n \in \mathbb{N}^{*}}$ and $\left\{R_{n, k}^{+}\right\}_{n \in \mathbb{N}^{*}}$ are of Følner-Tempelman type with respect to $\mathbb{G}_{k}$, applying Theorem 2.16 with $\Theta=\mathcal{S}\left(\mathbb{U}_{k}\right)$ we deduce that there exists $\widehat{\Omega} \in \mathcal{F}$ with $\mathbb{P}(\widehat{\Omega})=$

$$
\lim _{n \rightarrow \infty} \frac{\mathcal{A}_{k}\left(R_{n, k}^{-}\right)(\omega)}{\left|R_{n, k}^{-}\right|}=\lim _{n \rightarrow \infty} \frac{\mathcal{A}_{k}\left(R_{n, k}^{+}\right)(\omega)}{\left|R_{n, k}^{+}\right|}=\mathbb{E}^{\mathcal{I}_{\mathbb{G}_{k}}}\left[\mathcal{S}\left(\mathbb{U}_{k}\right)\right](\omega) \text { for all } \omega \in \widehat{\Omega} .
$$

On the other hand, by using (2.34) with $E=R_{n, k}^{+}$and (2.35) with $E=R_{n, k}^{-}$we get:

$$
\begin{aligned}
& \frac{\mathcal{S}\left(J_{n, k}^{+}\right)(\omega)}{\mu\left(J_{n, k}^{+}\right)}-\frac{1}{\mu\left(\mathbb{U}_{k}\right)} \frac{\mathcal{A}_{k}\left(R_{n, k}^{+}\right)(\omega)}{\left|R_{n, k}^{+}\right|}=\frac{\mathcal{S}_{k}\left(R_{n, k}^{+}\right)(\omega)}{\left|R_{n, k}^{+}\right|} \geq \inf _{q \in \mathbb{N}^{*}} \frac{\mathcal{S}_{k}\left(R_{q, k}^{+}\right)(\omega)}{\left|R_{q, k}^{+}\right|} ; \\
& \frac{\mathcal{S}\left(J_{n, k}^{-}\right)(\omega)}{\mu\left(J_{n, k}^{-}\right)}-\frac{1}{\mu\left(\mathbb{U}_{k}\right)} \frac{\mathcal{A}_{k}\left(R_{n, k}^{-}\right)(\omega)}{\left|R_{n, k}^{-}\right|} \leq 0
\end{aligned}
$$

\footnotetext{
${ }^{4}$ Lindenstrauss's ergodic theorem is established under the weaker condition that $\left\{R_{n}\right\}_{n \in \mathbb{N}}$. is a tempered Følner sequence (see $[29$, Definition 1.1$]$ and $[12, \S 2]$ for more details). The tempered Følner condition implies
the Følner-Tempelman the Følner-Tempelman condition, but the converse is not true (see $[29,12])$.
} 
for all $\omega \in \Omega$. Letting $n \rightarrow \infty$ we deduce that:

$$
\begin{aligned}
& \underline{l}_{k}(\omega)-\frac{\mathbb{E}^{\mathcal{I}_{\mathbb{G}_{k}}}\left[\mathcal{S}\left(\mathbb{U}_{k}\right)\right](\omega)}{\mu\left(\mathbb{U}_{k}\right)} \geq \inf _{n \in \mathbb{N}^{*}} \frac{\mathcal{S}_{k}\left(R_{n, k}^{+}\right)(\omega)}{\left|R_{n, k}^{+}\right|} \text {for all } k \in \mathbb{N}^{*} \text { and all } \omega \in \widehat{\Omega} ; \\
& \bar{l}_{k}(\omega)-\frac{\mathbb{E}^{\mathcal{I}_{\mathbb{G}_{k}}\left[\mathcal{S}\left(\mathbb{U}_{k}\right)\right](\omega)}}{\mu\left(\mathbb{U}_{k}\right)} \leq 0 \text { for all } k \in \mathbb{N}^{*} \text { and all } \omega \in \widehat{\Omega} ;
\end{aligned}
$$

In what follows, without loss of generality, we assume that $\widehat{\Omega}=\Omega$.

Substep 2-3: using a maximal inequality. We need the following lemma (see [12, Lemma 3.5] and also [3, Theorem 4.2]).

Lemma 2.17. Let $\mathcal{K}: \mathcal{P}_{\mathrm{f}}\left(\mathbb{G}_{k}\right) \rightarrow L^{1}(\Omega, \mathcal{F}, \mathbb{P})$ be a negative subadditive process and let $\left\{R_{n}\right\}_{n \in \mathbb{N}^{*}} \subset \mathcal{P}_{\mathrm{f}}\left(\mathbb{G}_{k}\right)$. Fix $\alpha>0$ and consider $V_{\alpha}^{\mathcal{K}} \in \mathcal{F}$ given by

$$
V_{\alpha}^{\mathcal{K}}:=\left\{\omega \in \Omega: \inf _{n \in \mathbb{N}^{*}} \frac{\mathcal{K}\left(R_{n}\right)(\omega)}{\left|R_{n}\right|}<-\alpha\right\} .
$$

If $\left\{R_{n}\right\}_{n \in \mathbb{N}^{*}}$ is of Følner-Tempelman type with respect to $\mathbb{G}_{k}$ then

$$
\mathbb{P}\left(V_{\alpha}^{\mathcal{K}}\right) \leq-\frac{M}{\alpha} \varlimsup_{n \rightarrow \infty} \frac{\mathbb{E}\left[\mathcal{K}\left(R_{n}\right)\right]}{\left|R_{n}\right|},
$$

where $M>0$ is the Templeman constant associated with $\left\{G_{n}\right\}_{n \in \mathbb{N}^{*}}$.

As $\mathcal{S}_{k}: \mathcal{P}_{\mathrm{f}}\left(\mathbb{G}_{k}\right) \rightarrow L^{1}(\Omega, \mathcal{F}, \mathbb{P})$ defined by (2.34) is a negative subadditive process, we can apply Theorem 2.17 with $\mathcal{K}=\mathcal{S}_{k}$. Hence, since $\left\{R_{n, k}^{+}\right\}_{n \in \mathbb{N}^{*}}$ is of Følner-Tempelman type with respect to $\mathbb{G}_{k}$, one has

$$
\mathbb{P}\left(V_{\alpha}^{\mathcal{S}_{k}}\right) \leq-\frac{M_{k}}{\alpha} \varlimsup_{n \rightarrow \infty} \frac{\mathbb{E}\left[\mathcal{S}_{k}\left(R_{n, k}^{+}\right)\right]}{\left|R_{n, k}^{+}\right|}
$$

where $M_{k}>0$ is the Templeman constant associated with $\left\{R_{n, k}^{+}\right\}_{n \in \mathbb{N}^{*}}$. Consequently, taking (2.38) into account, we obtain

$$
\mathbb{P}\left(V_{\alpha}^{\mathcal{S}_{k}}\right) \leq \frac{M_{k}}{\alpha} \varepsilon \text { for all } k \geq k_{\varepsilon}
$$

Substep 2-4: end of Step 2. From (2.41) and (2.42) it follows that

$$
\bar{l}_{k}-\underline{l}_{k} \leq-\inf _{n \in \mathbb{N}^{*}} \frac{\mathcal{S}_{k}\left(R_{n, k}^{+}\right)}{\left|R_{n, k}^{+}\right|} .
$$

Hence $W_{k, \alpha} \subset V_{\alpha}^{\mathcal{S}_{k}}$, where $W_{k, \alpha}$ is defined in (2.32). From (2.43) we conclude that (2.33) is satisfied with $k=k_{\varepsilon}$. 
In what follows we set $l:=\bar{l}=\underline{l}$ and $\gamma:=\inf _{k \in \mathbb{N}^{*}} \gamma_{k}$ with $\gamma_{k}:=\frac{\mathbb{E}_{\mathbb{G}_{k}}^{\mathcal{I}_{k}}\left[\mathcal{S}\left(\mathbb{U}_{k}\right)\right]}{\mu\left(\mathbb{U}_{k}\right)}$ for all $k \in \mathbb{N}^{*}$.

Step 3: we prove that $l=\gamma$. First of all, from (2.29) and (2.42) we see that $l(\omega) \leq \gamma_{k}(\omega)$ for $\mathbb{P}$-a.a. $\omega \in \Omega$ and all $k \in \mathbb{N}^{*}$, and so

$$
l(\omega) \leq \gamma(\omega) \text { for } \mathbb{P} \text {-a.a. } \omega \in \Omega
$$

On the other hand, letting $n \rightarrow \infty$ in (2.40) and using (2.39) we get

$$
\varlimsup_{n \rightarrow \infty} \frac{\mathcal{S}\left(J_{n, k}^{+}\right)(\omega)}{\mu\left(J_{n, k}^{+}\right)}-\gamma_{k}(\omega) \geq \varlimsup_{n \rightarrow \infty} \frac{\mathcal{S}_{k}\left(R_{n, k}^{+}\right)(\omega)}{\left|R_{n, k}^{+}\right|} \text {for } \mathbb{P} \text {-a.a. } \omega \in \Omega
$$

and so, taking (2.30) into account, one has

$$
l(\omega)-\gamma_{k} \geq \varlimsup_{n \rightarrow \infty} \frac{\mathcal{S}_{k}\left(R_{n, k}^{+}\right)(\omega)}{\left|R_{n, k}^{+}\right|} \text {for } \mathbb{P} \text {-a.a. } \omega \in \Omega .
$$

It follows that

$$
\int_{\Omega}\left[l(\omega)-\gamma_{k}\right] d \mathbb{P}(\omega) \geq \int_{\Omega} \varlimsup_{n \rightarrow \infty} \frac{\mathcal{S}_{k}\left(R_{n, k}^{+}\right)(\omega)}{\left|R_{n, k}^{+}\right|} d \mathbb{P}(\omega) .
$$

But, by using Fatou's lemma and (2.38) we see that for any $k \geq k_{\varepsilon}$, one has

$$
\int_{\Omega} \varlimsup_{n \rightarrow \infty} \frac{\mathcal{S}_{k}\left(R_{n, k}^{+}\right)(\omega)}{\left|R_{n, k}^{+}\right|} d \mathbb{P}(\omega)>-\varepsilon
$$

and consequently

$$
\begin{aligned}
\int_{\Omega} l(\omega) d \mathbb{P}(\omega) & \geq \int_{\Omega} \gamma_{k}(\omega) d \mathbb{P}(\omega)-\varepsilon \\
& \geq \int_{\Omega} \gamma(\omega) d \mathbb{P}(\omega)-\varepsilon .
\end{aligned}
$$

Letting $\varepsilon \rightarrow 0$ we deduce that

$$
\int_{\Omega}[l(\omega)-\gamma(\omega)] d \mathbb{P}(\omega) \geq 0
$$

and the result follows by combining (2.44) with (2.46).

In what follows, we set $\gamma^{\mathcal{I}}:=\inf _{k \in \mathbb{N}^{*}} \gamma_{k}^{\mathcal{I}}$ with $\gamma_{k}^{\mathcal{I}}:=\frac{\mathbb{E}^{\mathcal{I}}\left[\mathcal{S}\left(\mathbb{U}_{k}\right)\right]}{\mu\left(\mathbb{U}_{k}\right)}$ for all $k \in \mathbb{N}^{*}$. 
Step 4: we prove that $l=\gamma^{\mathcal{I}}$. Since $\gamma_{k}$ is $\mathcal{I}_{\mathbb{G}_{k}}$-measurable for all $k \in \mathbb{N}^{*}, \gamma=\inf _{k \in \mathbb{N}^{*}} \gamma_{k}$ is $\cap_{k \in \mathbb{N}^{*}} \mathcal{I}_{\mathbb{G}_{k}}$-measurable. But $\cap_{k \in \mathbb{N}^{*}} \mathcal{I}_{\mathbb{G}_{k}}=\mathcal{I}$ because $\cup_{k \in \mathbb{N}^{*}} \mathbb{G}_{k}=\mathbb{G}$, hence $\gamma$ is $\mathcal{I}$ measurable and so $l$ is $\mathcal{I}$-measurable by Step 3 . It follows that

$$
\mathbb{E}^{\mathcal{I}}[l]=l .
$$

As $\mathcal{I} \subset \mathcal{I}_{\mathbb{G}_{k}}$ for all $k \in \mathbb{N}^{*}$ we also have

$$
\mathbb{E}^{\mathcal{I}}\left[\gamma_{k}\right]=\gamma_{k}^{\mathcal{I}} \text { for all } k \in \mathbb{N}^{*}
$$

Arguing as in Step 3, for each $k \in \mathbb{N}^{*}$, we have $l \leq \gamma_{k}$ hence $\mathbb{E}^{\mathcal{I}}[l] \leq \mathbb{E}^{\mathcal{I}}\left[\gamma_{k}\right]$ and so $l \leq \gamma_{k}^{\mathcal{I}}$ by using (2.47) and (2.48). Consequently

$$
l \leq \gamma^{\mathcal{I}}
$$

Fix any $A \in \mathcal{I}$. Arguing again as in Step 3 we see that for any $k \geq k_{\varepsilon}$, one has

$$
\int_{A} l(\omega) d \mathbb{P}(\omega) \geq \int_{A} \gamma_{k}(\omega) d \mathbb{P}(\omega)-\varepsilon .
$$

But $\int_{A} \gamma_{k}(\omega) d \mathbb{P}(\omega)=\int_{A} \mathbb{E}^{\mathcal{I}}\left[\gamma_{k}\right](\omega) d \mathbb{P}(\omega)$ by definition of the conditional expectation, hence $\int_{A} \gamma_{k}(\omega) d \mathbb{P}(\omega)=\int_{A} \gamma_{k}^{\mathcal{I}}(\omega) d \mathbb{P}(\omega)$ by $(2.48)$, and so

$$
\begin{aligned}
\int_{A} l(\omega) d \mathbb{P}(\omega) & \geq \int_{A} \gamma_{k}^{\mathcal{I}}(\omega) d \mathbb{P}(\omega)-\varepsilon \\
& \geq \int_{A} \gamma^{\mathcal{I}}(\omega) d \mathbb{P}(\omega)-\varepsilon .
\end{aligned}
$$

Letting $\varepsilon \rightarrow 0$ we get

$$
\int_{A} l(\omega) d \mathbb{P}(\omega) \geq \int_{A} \gamma^{\mathcal{I}}(\omega) d \mathbb{P}(\omega) \text { for all } A \in \mathcal{I} \text {. }
$$

Combining (2.49) with (2.50) we deduce that

$$
\int_{A} l(\omega) d \mathbb{P}(\omega)=\int_{A} \gamma^{\mathcal{I}}(\omega) d \mathbb{P}(\omega) \text { for all } A \in \mathcal{I},
$$

which implies that $l=\mathbb{E}^{\mathcal{I}}\left[\gamma^{\mathcal{I}}\right]$ by unicity of the conditional expectation. But $\gamma^{\mathcal{I}}$ is $\mathcal{I}$-measurable because $\gamma_{k}^{\mathcal{I}}$ is $\mathcal{I}$-measurable for all $k \in \mathbb{N}^{*}$, hence $\mathbb{E}^{\mathcal{I}}\left[\gamma^{\mathcal{I}}\right]=\gamma^{\mathcal{I}}$ and conse-
quently $l=\gamma^{\mathcal{I}}$. 圆 
2.3. Extension to more general sequences of sets

Let $\mathcal{B} \subset \mathcal{M}$ be a $\mathbb{G}$-ring such that $\mathcal{B} \supset \mathcal{J}$, and let $\left\{J_{n}\right\}_{n \in \mathbb{N}^{*}} \subset \mathcal{B}$.

Definition 2.18. Assume that $(X, \mathcal{M}, \mu)$ is $\mathbb{G}$-meshable (resp. strongly $\mathbb{G}$-meshable). We say that $\left\{J_{n}\right\}_{n \in \mathbb{N}^{*}}$ is asymptotically $\mathbb{G}$-regular (resp. asymptotically strongly $\mathbb{G}$-regular) if there exist two $\mathbb{G}$-regular (resp. strongly $\mathbb{G}$-regular) sequences $\left\{J_{n}^{-}\right\}_{n \in \mathbb{N}^{*}},\left\{J_{n}^{+}\right\}_{n \in \mathbb{N}^{*}} \subset$ $\mathcal{J}_{0}$ such that:

$$
\begin{aligned}
& J_{n}^{-} \subset J_{n} \subset J_{n}^{+} \text {for all } n \in \mathbb{N}^{*} ; \\
& \lim _{n \rightarrow \infty} \frac{\mu\left(J_{n}^{+} \backslash J_{n}^{-}\right)}{\mu\left(J_{n}\right)}=0 .
\end{aligned}
$$

Theorems 2.5 and 2.14 can be extended to the class of sequences of sets characterized by Definition 2.18 .

Theorem 2.19. Assume that $(X, \mathcal{M}, \mu)$ is $\mathbb{G}$-meshable (resp. strongly $\mathbb{G}$-meshable) and consider $\mathcal{S}: \mathcal{B} \rightarrow \mathbb{R}$ a subadditive and $\mathbb{G}$-invariant set function (resp. $\mathcal{S}: \mathcal{B} \rightarrow$ $L^{1}(\Omega, \mathcal{F}, \mathbb{P})$ a subadditive process) satisfying $(2.3)$. If $\left\{J_{n}\right\}_{n \in \mathbb{N}^{*}}$ is asymptotically $\mathbb{G}$ regular (resp. asymptotically strongly $\mathbb{G}$-regular) then the conclusion of Theorem 2.5 resp. Theorem 2.14) holds.

Proof of Theorem 2.19. It suffices to prove that

$$
\varliminf_{n \rightarrow \infty} \frac{\mathcal{S}\left(J_{n}^{+}\right)}{\mu\left(J_{n}^{+}\right)} \leq \varliminf_{n \rightarrow \infty} \frac{\mathcal{S}\left(J_{n}\right)}{\mu\left(J_{n}\right)} \leq \varlimsup_{n \rightarrow \infty} \frac{\mathcal{S}\left(J_{n}\right)}{\mu\left(J_{n}\right)} \leq \varlimsup_{n \rightarrow \infty} \frac{\mathcal{S}\left(J_{n}^{-}\right)}{\mu\left(J_{n}^{-}\right)},
$$

and to apply Theorem 2.5 (resp. Theorem 2.14). The inequalities in (2.51) follow by the same approximation argument as in Step 2 of the proof of Theorem 2.5.

\section{Toward homogenization in Cheeger-Sobolev spaces}

3.1. Cheeger-Sobolev spaces

Let $(M, d, \mu)$ be a separable and complete metric measure space. In what follows, for each $x \in M$ and each $\rho>0$, the open ball centered at $x$ with radius $\rho$ is denoted by $B_{\rho}(x)$, and for each open set $O \subset M, \mathcal{O}(O)$ denotes the class of open subsets of $O$.

Here and subsequently, we assume that $\mu$ is doubling on $M$, i.e. there exists a constant $C_{d} \geq 1$ such that

$$
\mu\left(B_{\rho}(x)\right) \leq C_{d} \mu\left(B_{\frac{\rho}{2}}(x)\right)
$$

for $\mu$-a.a. $x \in M$ and all $\rho>0$, and $M$ supports a weak $(1, p)$-Poincaré inequality with $p>1$, i.e. there exist $C_{P}>0$ and $\sigma \geq 1$ such that for $\mu$-a.e. $x \in M$ and every $\rho>0$, 


$$
f_{B_{\rho}(x)}\left|u-f_{B_{\rho}(x)} u d \mu\right| d \mu \leq \rho C_{P}\left(f_{B_{\sigma \rho}(x)} v^{p} d \mu\right)^{\frac{1}{p}}
$$

for every $u \in L_{\mu}^{p}(O)$, every $p$-weak upper gradient ${ }^{5} v \in L_{\mu}^{p}(O)$ for $u$ and every $O \in \mathcal{O}(M)$ such that $B_{\sigma \rho}(x) \subset O$. We further assume that $(M, d, \mu)$ satisfies the annular decay property, i.e. there exist $\delta>0$ and $C_{A} \geq 1$ such that

$$
\mu\left(B_{\sigma r}(x) \backslash B_{r}(x)\right) \leq C_{A}\left(1-\frac{1}{\sigma}\right)^{\delta} \mu\left(B_{\sigma r}(x)\right)
$$

for all $x \in M$, all $r>0$ and all $\sigma \in] 1, \infty[$.

Remark 3.1. From [6, Corollary 2.2] and [9, Lemma 3.3] (see also [8, Proposition 6.12] and [22, Proposition 11.5.3 pp. 328]), under (3.1) and (3.2), if moreover $(M, d)$ is a length space, i.e. the distance between any two points equals infimum of lengths of curves connecting the points, then (3.3) holds.

Remark 3.2. If (3.3) holds then $\mu\left(\overline{B_{r}(x)} \backslash B_{r}(x)\right)=0$ for all $x \in M$ and all $r>0$, i.e. the boundary of balls is of zero measure. Indeed, given $x \in M$ and $r>0$, we have $1 \geq \mu\left(B_{r}(x)\right) / \mu\left(\overline{B_{r}(x)}\right) \geq \mu\left(B_{r}(x)\right) / \mu\left(B_{\sigma r}(x)\right) \geq 1-C_{A}\left(1-\frac{1}{\sigma}\right)^{\delta}$ for all $\left.\sigma \in\right] 1, \infty[$. Hence, by letting $\sigma \rightarrow 1$, we obtain $\mu\left(B_{r}(x)\right) / \mu\left(\overline{B_{r}(x)}\right)=1$, i.e. $\mu\left(B_{r}(x)\right)=\mu\left(\overline{B_{r}(x)}\right)$.

Let $O \in \mathcal{O}(M)$ be bounded. Denote the algebra of Lipschitz functions from $O$ to $\mathbb{R}$ by Lip $(O)$. (Note that, by Hopf-Rinow's theorem (see [4, Proposition 3.7, pp. 35]), the closure of $O$ is compact, and so every Lipschitz function from $O$ to $\mathbb{R}$ is bounded.) Let $\operatorname{Lip}\left(O ; \mathbb{R}^{m}\right):=[\operatorname{Lip}(\mathrm{O})]^{m}$ and let $\nabla_{\mu}: \operatorname{Lip}\left(O ; \mathbb{R}^{m}\right) \rightarrow L_{\mu}^{\infty}(O ; \mathbb{M})$ be given by

$$
\nabla_{\mu} u:=\left(\begin{array}{c}
D_{\mu} u_{1} \\
\vdots \\
D_{\mu} u_{m}
\end{array}\right) \text { with } u=\left(u_{1}, \cdots, u_{m}\right)
$$

where $D_{\mu}: \operatorname{Lip}(O) \rightarrow L_{\mu}^{\infty}\left(O ; \mathbb{R}^{N}\right)$ is the differential of Cheeger (see [8, Theorem 4.38] and [24, Definition 2.1.1 and Theorem 2.3.1] for more details). The $p$-Cheeger-Sobolev space $H_{\mu}^{1, p}\left(O ; \mathbb{R}^{m}\right)$ is defined as the completion of $\operatorname{Lip}\left(O ; \mathbb{R}^{m}\right)$ with respect to the norm

$$
\|u\|_{H_{\mu}^{1, p}\left(O ; \mathbb{R}^{m}\right)}:=\|u\|_{L_{\mu}^{p}\left(O ; \mathbb{R}^{m}\right)}+\left\|\nabla_{\mu} u\right\|_{L_{\mu}^{p}(O ; \mathbb{M})}
$$

\footnotetext{
${ }^{5}$ A Borel function $v: O \rightarrow[0, \infty]$ is said to be an upper gradient for $u: O \rightarrow \mathbb{R}$ if $|u(c(1))-u(c(0))| \leq$ $\int_{0}^{1} v(c(s)) d s$ for all continuous rectifiable curves $c:[0,1] \rightarrow O$. A function $v \in L_{\mu}^{p}(O)$ is said to be a $p$-weak upper gradient for $u \in L_{\mu}^{p}(O)$ if there exist $\left\{u_{n}\right\}_{n \in \mathbb{N}} \cdot \subset L_{\mu}^{p}(O)$ and $\left\{v_{n}\right\}_{n \in \mathbb{N}} \cdot \mathcal{C}^{\mu} L_{\mu}^{p}(O)$ such that for each $n \in \mathbb{N}^{*}, v_{n}$ is an upper gradient for $u_{n}, u_{n} \rightarrow u$ in $L_{\mu}^{p}(O)$ and $v_{n} \rightarrow v$ in $L_{\mu}^{p}(O)$. For more details we refer to $[21,8]$.
} 
As $\left\|\nabla_{\mu} u\right\|_{L_{\mu}^{p}(O ; \mathbb{M})} \leq\|u\|_{W_{\mu}^{1, p}\left(O ; \mathbb{R}^{m}\right)}$ for all $u \in \operatorname{Lip}\left(O ; \mathbb{R}^{m}\right)$, the linear map $\nabla_{\mu}$ from $\operatorname{Lip}\left(O ; \mathbb{R}^{m}\right)$ to $L_{\mu}^{p}(O ; \mathbb{M})$ has a unique extension to $H_{\mu}^{1, p}\left(O ; \mathbb{R}^{m}\right)$ which will still be denoted by $\nabla_{\mu}$ and will be called the $\mu$-gradient. For more details on the various possible extensions of the classical theory of the Sobolev spaces to the setting of metric measure spaces, we refer to $[20, \S 10-14]$ (see also $[8,38,18,19]$ ).

\section{2. Г-convergence}

Let $p>1$ and let $(\Omega, \mathcal{F}, \mathbb{P})$ be a probability space. For each $n \in \mathbb{N}^{*}$, we consider a variational stochastic integral $E_{n}: H_{\mu}^{1, p}\left(O ; \mathbb{R}^{m}\right) \times \mathcal{O}(O) \times \Omega \rightarrow[0, \infty]$ defined by

$$
E_{n}(u, A, \omega):=\int_{A} L_{n}\left(x, \nabla_{\mu} u(x), \omega\right) d \mu(x),
$$

where $L_{n}: O \times \mathbb{M} \times \Omega \rightarrow[0, \infty]$ is a Borel measurable stochastic integrand ${ }^{6}$ depending on $n \in \mathbb{N}^{*}$, not necessarily convex with respect to $\xi \in \mathbb{M}$, where $\mathbb{M}$ denotes the space of real $m \times N$ matrices, and having $p$-growth, i.e. there exist $\alpha, \beta>0$, which do not depend
on $n$, such that

$$
\alpha|\xi|^{p} \leq L_{n}(x, \xi, \omega) \leq \beta\left(1+|\xi|^{p}\right)
$$

for all $x \in O$, all $\xi \in \mathbb{M}$ and $\mathbb{P}$-a.a. $\omega \in \Omega$. It is of interest to deal with the problem of computing the almost sure $\Gamma$-convergence with respect to the strong convergence of $L_{\mu}^{p}\left(O ; \mathbb{R}^{m}\right)$ (see Definitions 3.3 and 3.4) of $\left\{E_{n}\right\}_{n \in \mathbb{N}^{*}}$ as $n \rightarrow \infty$ toward a variational stochastic integral $E_{\infty}: H_{\mu}^{1, p}\left(O ; \mathbb{R}^{m}\right) \times \mathcal{O}(O) \times \Omega \rightarrow[0, \infty]$ of the type

$$
E_{\infty}(u, A, \omega)=\int_{A} L_{\infty}\left(x, \nabla_{\mu} u(x), \omega\right) d \mu(x)
$$

with $L_{\infty}: O \times \mathbb{M} \times \Omega \rightarrow[0, \infty]$ not depending on $n$. When $L_{\infty}$ is independent of the variable $x$, the procedure of passing from (3.4) to (3.6) is referred as stochastic homogenization. If furthermore $L_{\infty}$ is independent of the variable $\omega$ then $E_{\infty}$ is said to be deterministic. When $\left\{L_{n}\right\}_{n \in \mathbb{N} *}$ is deterministic, i.e. $L_{n}$ is independent of the variable $\omega$ for all $n \in \mathbb{N}^{*}$, the procedure of passing from (3.4) to (3.6) is referred as deterministic homogenization. Deterministic and stochastic homogenization were studied by many authors in the Euclidean case, i.e. when $(M, d, \mu)=\left(\mathbb{R}^{N},|\cdot-\cdot|, \mathcal{L}_{N}\right)$ where $\mathcal{L}_{N}$ is the Lebesgue measure on $\mathbb{R}^{N}$, see for instance [11] and the references therein.

Let us recall the definition of $\Gamma$-convergence and almost sure $\Gamma$-convergence. For more details on the theory of $\Gamma$-convergence we refer to [13].

\footnotetext{
${ }^{6}$ By a Borel measurable stochastic integrand $L: O \times \mathbb{M} \times \Omega \rightarrow[0, \infty]$ we mean that $L$ is $(\mathcal{B}(M) \otimes$ respectively.
} 
Definition 3.3. For each $n \in \mathbb{N}^{*}$, let $E_{n}: H_{\mu}^{1, p}\left(O ; \mathbb{R}^{m}\right) \times \mathcal{O}(O) \rightarrow[0, \infty]$ and let $E_{\infty}$ : $H_{\mu}^{1, p}\left(O ; \mathbb{R}^{m}\right) \times \mathcal{O}(O) \rightarrow[0, \infty]$. We say that $\left\{E_{n}\right\}_{n \in \mathbb{N}^{*}} \Gamma$-converges with respect to the strong convergence of $L_{\mu}^{p}\left(O ; \mathbb{R}^{m}\right)$, or simply $\Gamma\left(L_{\mu}^{p}\right)$-converges, to $E_{\infty}$ as $n \rightarrow \infty$ if

$$
\Gamma\left(L_{\mu}^{p}\right)-\varliminf_{n \rightarrow \infty} E_{n}(u, A) \geq E_{\infty}(u, A) \geq \Gamma\left(L_{\mu}^{p}\right)-\varlimsup_{n \rightarrow \infty} E_{n}(u, A)
$$

for all $u \in H_{\mu}^{1, p}\left(O ; \mathbb{R}^{m}\right)$ and all $A \in \mathcal{O}(O)$, with:

$$
\begin{aligned}
& \Gamma\left(L_{\mu}^{p}\right)-\varliminf_{n \rightarrow \infty} E_{n}(u, A):=\inf \left\{\varliminf_{n \rightarrow \infty} E_{n}\left(u_{n}, A\right): u_{n} \stackrel{L_{\mu}^{p}}{\rightarrow} u\right\} ; \\
& \Gamma\left(L_{\mu}^{p}\right)-\varlimsup_{n \rightarrow \infty} E_{n}(u, A):=\inf \left\{\varlimsup_{n \rightarrow \infty} E_{n}\left(u_{n}, A\right): u_{n} \stackrel{L_{\mu}^{p}}{\rightarrow} u\right\} .
\end{aligned}
$$

Then we write

$$
\Gamma\left(L_{\mu}^{p}\right)-\lim _{n \rightarrow \infty} E_{n}(u, A)=E_{\infty}(u, A)
$$

Almost sure $\Gamma$-convergence is defined from Definition 3.3 as follows.

Definition 3.4. For each $n \in \mathbb{N}^{*}$, let $E_{n}: H_{\mu}^{1, p}\left(O ; \mathbb{R}^{m}\right) \times \mathcal{O}(O) \times \Omega \rightarrow[0, \infty]$ and let $E_{\infty}: H_{\mu}^{1, p}\left(O ; \mathbb{R}^{m}\right) \times \mathcal{O}(O) \times \Omega \rightarrow[0, \infty]$. We say that $\left\{E_{n}\right\}_{n \in \mathbb{N}^{*}}$ almost sure $\Gamma$-converges with respect to the strong convergence of $L_{\mu}^{p}\left(O ; \mathbb{R}^{m}\right)$, or simply almost sure $\Gamma\left(L_{\mu}^{p}\right)$ converges, to $E_{\infty}$ as $n \rightarrow \infty$ if for $\mathbb{P}$-a.e. $\omega \in \Omega$, one has

$$
\Gamma\left(L_{\mu}^{p}\right)-\lim _{n \rightarrow \infty} E_{n}(u, A, \omega)=E_{\infty}(u, A, \omega)
$$

for all $u \in H_{\mu}^{1, p}\left(O ; \mathbb{R}^{m}\right)$ and all $A \in \mathcal{O}(O)$.

For each $n \in \mathbb{N}^{*}$ and each $\rho>0$, let $\mathcal{H}_{\mu}^{\rho} L_{n}: O \times \mathbb{M} \times \Omega \rightarrow[0, \infty]$ be given by

$$
\mathcal{H}_{\mu}^{\rho} L_{n}(x, \xi, \omega):=\inf \left\{\int_{B_{\rho}(x)} L_{n}\left(y, \xi+\nabla_{\mu} w(y), \omega\right) d \mu(y): w \in H_{\mu, 0}^{1, p}\left(B_{\rho}(x) ; \mathbb{R}^{m}\right)\right\}
$$

where the space $H_{\mu, 0}^{1, p}\left(B_{\rho}(x) ; \mathbb{R}^{m}\right)$ is the closure of

$$
\operatorname{Lip}_{0}\left(B_{\rho}(x) ; \mathbb{R}^{m}\right):=\left\{u \in \operatorname{Lip}\left(O ; \mathbb{R}^{m}\right): u=0 \text { on } O \backslash B_{\rho}(x)\right\}
$$

with respect to the $H_{\mu}^{1, p}$-norm. When $\left\{L_{n}\right\}_{n \in \mathbb{N}^{*}}$ is deterministic, in [2, Theorem 2.2 and Remark 4.2] we proved the following result.

Theorem 3.5. If (3.5) holds then for every $u \in H_{\mu}^{1, p}\left(O ; \mathbb{R}^{m}\right)$, the set function

$$
\Gamma\left(L_{\mu}^{p}\right)-\varliminf_{n \rightarrow \infty} E_{n}(u, \cdot): \mathcal{O}(O) \rightarrow[0, \infty]
$$


can be uniquely extended to a finite positive Radon measure on $O$ which is absolutely continuous with respect to $\mu$, and:

$$
\begin{aligned}
& \Gamma\left(L_{\mu}^{p}\right)-\varliminf_{n \rightarrow \infty} E_{n}(u, A) \geq \int_{A} \varlimsup_{\rho \rightarrow 0} \varliminf_{n \rightarrow \infty} \mathcal{H}_{\mu}^{\rho} L_{n}\left(x, \nabla_{\mu} u(x)\right) d \mu(x) ; \\
& \Gamma\left(L_{\mu}^{p}\right)-\varlimsup_{n \rightarrow \infty} E_{n}(u, A)=\int_{A} \lim _{\rho \rightarrow 0} \varlimsup_{n \rightarrow \infty} \mathcal{H}_{\mu}^{\rho} L_{n}\left(x, \nabla_{\mu} u(x)\right) d \mu(x)
\end{aligned}
$$

for all $A \in \mathcal{O}(O)$.

By the same method we can establish the following stochastic version of Theorem 3.5.

Theorem 3.6. If (3.5) holds then for every $u \in H_{\mu}^{1, p}\left(O ; \mathbb{R}^{m}\right)$ and $\mathbb{P}$-a.e. $\omega \in \Omega$, the set
function

$$
\Gamma\left(L_{\mu}^{p}\right)-\varliminf_{n \rightarrow \infty} E_{n}(u, \cdot, \omega): \mathcal{O}(O) \rightarrow[0, \infty]
$$

can be uniquely extended to a finite positive Radon measure on $O$ which is absolutely continuous with respect to $\mu$, and:

$$
\begin{aligned}
& \Gamma\left(L_{\mu}^{p}\right)-\varliminf_{n \rightarrow \infty} E_{n}(u, A, \omega) \geq \int_{A} \varlimsup_{\rho \rightarrow 0} \varliminf_{n \rightarrow \infty} \mathcal{H}_{\mu}^{\rho} L_{n}\left(x, \nabla_{\mu} u(x), \omega\right) d \mu(x) ; \\
& \Gamma\left(L_{\mu}^{p}\right)-\varlimsup_{n \rightarrow \infty} E_{n}(u, A, \omega)=\int_{A} \lim _{\rho \rightarrow 0} \varlimsup_{n \rightarrow \infty} \mathcal{H}_{\mu}^{\rho} L_{n}\left(x, \nabla_{\mu} u(x), \omega\right) d \mu(x)
\end{aligned}
$$

for all $A \in \mathcal{O}(O)$.

\subsection{Homogenization}

Homogenization of integrals of the calculus of variations in non-euclidean settings has been studied for the first time in the recent paper [2] (see also [32]). Although extending results available for Sobolev spaces to Cheeger-Sobolev spaces is of interest (especially from the point of view of hyperelasticity where the support of the measure $\mu$ can be interpreted as a mechanical structure together with its singularities like for example thin dimensions, corners, junctions, etc.) this subject is still at the beginning and its development needs further investigations.

In this paragraph, we develop an abstract framework to deal with homogenization of integrals of type (3.4) by using subadditive theorems established in Sect. 2. We restrict our attention to the stochastic case (see Theorem 3.12). The deterministic case can be treated by the same method. 
Let $I$ be a finite set and let $\left\{M_{i}\right\}_{i \in I} \subset \mathcal{B}(M)$ where $\mathcal{B}(M)$ the Borel subsets of $(M, d)$. From now on, we assume that:

$$
\begin{aligned}
& M=\underset{i \in I}{\cup} M_{i} \text { with } \mu\left(M_{i} \cap M_{j}\right)=0 \text { for all } i, j \in I \text { such that } i \neq j ; \\
& \mathcal{O}\left(M_{i} \backslash N_{i}\right) \subset \mathcal{O}(M) \text { for all } i \in I,
\end{aligned}
$$
where $N_{i}:=\cup_{j \neq i} M_{i} \cap M_{j}$. Setting $O_{i}:=\left(M_{i} \backslash N_{i}\right) \cap O$ for all $i \in I$, from (3.7) and (3.8)
we have:

$$
\begin{aligned}
& \mu\left(O_{i} \cap O_{j}\right)=0 \text { for all } i, j \in I \text { such that } i \neq j ; \\
& \mu\left(O \backslash \bigcup_{i \in I} O_{i}\right)=0 ; \\
& O_{i} \in \mathcal{O}(O) \text { for all } i \in I .
\end{aligned}
$$

Remark 3.7. From (3.7) we have $\mu\left(M_{i} \cap M_{j}\right)=0$ for all $i, j \in I$ such that $i \neq j$, and so $\mu\left(M_{i}\right)=\mu\left(M_{i} \backslash N_{i}\right)$ and $\mu\left(O_{i}\right)=\mu\left(M_{i} \cap O\right)$ for all $i \in I$.

For each $i \in I$, let $\left(\Omega_{i}, \mathcal{F}_{i}, \mathbb{P}_{i}\right)$ be a probability space and let $L^{i}: M_{i} \times \mathbb{M} \times \Omega_{i} \rightarrow[0, \infty]$ be a Borel measurable stochastic integrand having $p$-growth, i.e. there exist $\alpha, \beta>0$,
not depending on $i$, such that

$$
\alpha|\xi|^{p} \leq L^{i}\left(x, \xi, \omega_{i}\right) \leq \beta\left(1+|\xi|^{p}\right)
$$

for all $x \in M_{i}$, all $\xi \in \mathbb{M}$ and $\mathbb{P}_{i}$-a.a. $\omega_{i} \in \Omega_{i}$. Let $\mathcal{B}\left(M_{i}\right)$ be the Borel subsets of $\left(M_{i}, d\right)$. As $M_{i} \in \mathcal{B}(M)$ we can assert that $\mathcal{B}\left(M_{i}\right) \subset \mathcal{B}(M)$ and so $\left(M_{i}, \mathcal{B}\left(M_{i}\right), \mu\right)$ is a measure space. In what follows, we adopt the following notation:

- we denote by $\mathcal{B}_{0}^{i}$ the class of $A \in \mathcal{B}\left(M_{i}\right)$ such that $\mu(A)<\infty$ and $\mu(\partial A)=0$ with $\partial A=\bar{A} \backslash \AA$, where $\bar{A}$ (resp. $\AA$ ) is the closure (resp. the interior) of $A$;

- we denote by $\operatorname{Homeo}\left(M_{i}\right)$ the group of homeomorphism on $M_{i}$;

- we denote by $\mathrm{Ba}\left(M_{i} \backslash N_{i}\right)$ the class of open ball of $M_{i} \backslash N_{i}$.

Remark 3.8. As $\mathrm{Ba}\left(M_{i} \backslash N_{i}\right) \subset \mathcal{O}(M)$ by (3.8), from Remark 3.2 we see that $\mu(\partial B)=0$ for all $B \in \mathrm{Ba}\left(M_{i} \backslash N_{i}\right)$, and so $\mathrm{Ba}\left(M_{i} \backslash N_{i}\right) \subset \mathcal{B}_{0}^{i}$.

To extend homogenization to non-euclidean situations, it is necessary to make some refinements $^{7}$ on our general metric measure setting. For this, we consider:

- $\mathbb{G}_{i}$ a subgroup of $\operatorname{Homeo}\left(M_{i}\right)$;

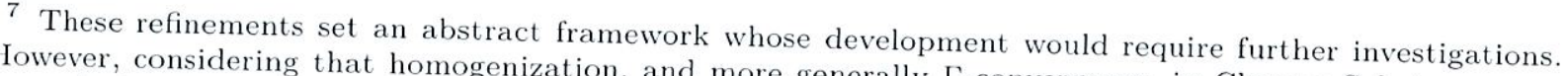
is at the beginning of its development, it seems of interest to present it.
} 
- $\left\{\tau_{g}^{i}\right\}_{g \in \mathbb{G}_{i}}$ a group of $\mathbb{P}_{i}$-preserving transformation on $\left(\Omega_{i}, \mathcal{F}_{i}, \mathbb{P}_{i}\right)$ (see Definition 2.10);

- $\left\{h_{n}^{i}\right\}_{n \in \mathbb{N}^{*}} \subset \operatorname{Homeo}\left(M_{i}\right)$;

- $\mathbb{U}_{i} \in \mathcal{B}_{0}^{i}$ such that $\mu\left(\mathbb{U}_{i}\right)>0$,

where $\mathbb{U}_{i}$ can be interpreted as the "unit cell" with respect to $M_{i}$ and $\left\{h_{n}^{i}\right\}_{n \in \mathbb{N} *}$ as "dilations" in $M_{i}$, and we assume that:

$\left(\mathrm{H}_{0}^{i}\right) L^{i}\left(g^{-1}(x), \xi, \omega_{i}\right)=L^{i}\left(x, \xi, \tau_{g}^{i}\left(\omega_{i}\right)\right)$ for all $x \in M_{i}$, all $\xi \in \mathbb{M}$, all $g \in \mathbb{G}_{i}$ and $\mathbb{P}_{i}$-a.a. $\omega_{i} \in \Omega_{i}$; $\left(\mathrm{H}_{1}^{i}\right)\left(M_{i}, \mathcal{B}\left(M_{i}\right), \mu\right)$ is strongly $\mathbb{G}_{i}$-meshable with respect to $\left\{h_{k}^{i}\left(\mathbb{U}_{i}\right)\right\}_{k \in \mathbb{N}^{*}}$ (see Defini-
tions 2.1 and 2.8);

$\left(\mathrm{H}_{2}^{i}\right) \mu$ is $\mathbb{G}_{i}$-invariant, i.e. $g^{\sharp} \mu=\mu$ for all $g \in \mathbb{G}_{i}$ which means that $\mu\left(g^{-1}(A)\right)=\mu(A)$ for all $g \in \mathbb{G}_{i}$ and all $A \in \mathcal{B}\left(M_{i}\right)$;

$\left(\mathrm{H}_{3}^{i}\right)$ for each $n \in \mathbb{N}^{*},\left(\left(h_{n}^{i}\right)^{-1}\right)^{\sharp} \mu=\mu\left(h_{n}^{i}\left(\mathbb{U}_{i}\right)\right) \mu$, i.e. $\mu\left(h_{n}^{i}(A)\right)=\mu\left(h_{n}^{i}\left(\mathbb{U}_{i}\right)\right) \mu(A)$ for all $A \in \mathcal{B}\left(M_{i}\right)$;

$\left(\mathrm{H}_{4}^{i}\right)$ for each $g \in \mathbb{G}_{i}$ and each $B \in \mathrm{Ba}\left(M_{i} \backslash N_{i}\right)$, there exists a bijective map $T_{g, B}^{i}$ from $H_{\mu, 0}^{1, p}\left(g^{-1}(B) ; \mathbb{R}^{m}\right)$ to $H_{\mu, 0}^{1, p}\left(B ; \mathbb{R}^{m}\right)$ such that $\nabla_{\mu} T_{g, B}^{i}(w)=\nabla_{\mu} w \circ g^{-1}$ for all $w \in H_{\mu, 0}^{1, p}\left(g^{-1}(B) ; \mathbb{R}^{m}\right)$ and $\nabla_{\mu}\left(T_{g, B}^{i}\right)^{-1}(v)=\nabla_{\mu} v \circ g$ for all $v \in H_{\mu, 0}^{1, p}\left(B ; \mathbb{R}^{m}\right)$;

$\left(\mathrm{H}_{5}^{i}\right)$ for each $n \in \mathbb{N}^{*}$ and each $B \in \operatorname{Ba}\left(M_{i} \backslash N_{i}\right)$, there exists a bijective map $H_{n, B}^{i}$ from $H_{\mu, 0}^{1, p}\left(h_{n}^{i}(B) ; \mathbb{R}^{m}\right)$ to $H_{\mu, 0}^{1, p}\left(B ; \mathbb{R}^{m}\right)$ such that $\nabla_{\mu} H_{n, B}^{i}(w)=\nabla_{\mu} w o h_{n}^{i}$ for all $w \in H_{\mu, 0}^{1, p}\left(h_{n}^{i}(B) ; \mathbb{R}^{m}\right)$ and $\nabla_{\mu}\left(H_{n, B}^{i}\right)^{-1}(v)=\nabla_{\mu} v o\left(h_{n}^{i}\right)^{-1}$ for all $v \in H_{\mu, 0}^{1, p}\left(B ; \mathbb{R}^{m}\right)$;

Remark 3.9. As $\mathbb{G}_{i} \subset \operatorname{Homeo}\left(M_{i}\right)$, from $\left(\mathrm{H}_{2}^{i}\right)$ we see that $\mathcal{B}_{0}^{i}$ is a $\mathbb{G}_{i}$-ring, i.e. if $A \in \mathcal{B}_{0}^{i}$ and if $g \in \mathbb{G}_{i}$ then $g^{-1}(A) \in \mathcal{B}_{0}^{i}$.

Remark 3.10. From $\left(H_{3}^{i}\right)$ we see that $\mu\left(h_{n}^{i}\left(\mathbb{U}_{i}\right)\right)>0$ and $\left(h_{n}^{i}\right)^{\sharp} \mu=\frac{1}{\mu\left(h_{n}^{i}\left(\mathbb{U}_{i}\right)\right)} \mu$ for all
$n \in \mathbb{N}^{*}$.

Remark 3.11. As $\mu\left(\overline{\mathbb{U}}_{i} \backslash \mathbb{U}_{i}\right)=0$ we have $\mu\left(\mathbb{U}_{i}\right)=\mu\left(\mathbb{U}_{i}\right)$ and, under $\left(\mathrm{H}_{3}^{i}\right)$, for each $n \in \mathbb{N}^{*}, \mu\left(h_{n}^{i}\left(\mathbb{U}_{i}\right)\right)=\mu\left(h_{n}^{i}\left(\mathbb{U}_{i}\right)\right)$ because $h_{n}^{i} \in \operatorname{Homeo}\left(M_{i}\right)$ and $\left(h_{n}^{i}\right)^{*} \mu=\frac{1}{\mu\left(h_{n}^{i}\left(\mathbb{U}_{i}\right)\right)} \mu$.

Let $\mathcal{J}_{0}^{i} \subset \mathcal{B}\left(M_{i}\right)$ be given by

$$
\mathcal{J}_{0}^{i}:=\left\{\bigcup_{g \in H} g^{-1}\left(h_{k}^{i}\left(\mathbb{U}_{i}\right)\right): k \in \mathbb{N}^{*}, H \in \mathcal{D}\left(h_{k}^{i}\left(\mathbb{U}_{i}\right)\right) \text { and }|H|<\infty\right\}
$$

with

$$
\mathcal{D}\left(h_{k}^{i}\left(\mathbb{U}_{i}\right)\right):=\left\{H \subset \mathbb{G}_{i}:\left\{g^{-1}\left(h_{k}^{i}\left(\mathbb{U}_{i}\right)\right)\right\}_{g \in H} \text { is disjoint }\right\},
$$

and let $\mathcal{J}^{i}$ be the $\mathbb{G}_{i}$-ring generated by $\mathcal{J}_{0}^{i}$. It is clear that $\mathcal{J}_{0}^{i} \subset \mathcal{B}_{0}^{i}$ and so $\mathcal{B}_{0}^{i} \supset \mathcal{J}^{i}$ because $\mathcal{B}_{0}^{i}$ is a $\mathbb{G}_{i}$-ring (see Remark 3.9). We further suppose that: 
$\left(\mathrm{H}_{6}^{i}\right)$ for each $B \in \mathrm{Ba}\left(M_{i} \backslash N_{i}\right),\left\{h_{n}^{i}(B)\right\}_{n \in \mathbb{N}^{*}}$ is asymptotically strongly $\mathbb{G}_{i}$-regular, i.e. there exist two strongly $\mathbb{G}_{i}$-regular sequences $\left\{J_{n}^{-}\right\}_{n \in \mathbb{N}^{*}},\left\{J_{n}^{+}\right\}_{n \in \mathbb{N}^{*}} \subset \mathcal{J}_{0}^{i}$ such that:

$$
\begin{aligned}
& J_{n}^{-} \subset h_{n}^{i}(B) \subset J_{n}^{+} \text {for all } n \in \mathbb{N}^{*} ; \\
& \lim _{n \rightarrow \infty} \frac{\mu\left(J_{n}^{+} \backslash J_{n}^{-}\right)}{\mu\left(h_{n}^{i}(B)\right)}=0 .
\end{aligned}
$$

(see Definitions 2.9 and 2.18).

Let $(\Omega, \mathcal{F}, \mathbb{P})$ be the product of the probability spaces $\left\{\left(\Omega_{i}, \mathcal{F}_{i}, \mathbb{P}_{i}\right)\right\}_{i \in I}$, i.e. $\Omega=$ $\prod_{i \in I} \Omega_{i}, \mathcal{F}=\otimes_{i \in I} \mathcal{F}_{i}$ and $\mathbb{P}=\otimes_{i \in I} \mathbb{P}_{i}$, and let $L: M \times \mathbb{M} \times \Omega \rightarrow[0, \infty]$ be defined by

$$
L(x, \xi, \omega)=L^{i}\left(x, \xi, \omega_{i}\right) \text { if } x \in M_{i}
$$

For each $n \in \mathbb{N}^{*}$, we define $h_{n}: M \rightarrow M$ by $h_{n}(x)=h_{n}^{i}(x)$ if $x \in M_{i}$ and we consider $L_{n}: O \times \mathbb{M} \times \Omega \rightarrow[0, \infty]$ given by

$$
L_{n}(x, \xi, \omega)=L\left(h_{n}(x), \xi, \omega\right)
$$

The following homogenization theorem is a consequence of Theorems 2.19 and 3.6.

Theorem 3.12. If $\left(\mathrm{H}_{0}^{i}\right)$, $\left(\mathrm{H}_{1}^{i}\right)$, $\left(\mathrm{H}_{2}^{i}\right)$, $\left(\mathrm{H}_{3}^{i}\right),\left(\mathrm{H}_{4}^{i}\right),\left(\mathrm{H}_{5}^{i}\right)$ and $\left(\mathrm{H}_{6}^{i}\right)$ hold for all $i \in I$, then for every $u \in H_{\mu}^{1, p}\left(O ; \mathbb{R}^{m}\right)$ and $\mathbb{P}$-a.e. $\omega \in \Omega$, one has

$$
\Gamma\left(L_{\mu}^{p}\right)-\lim _{n \rightarrow \infty} E_{n}(u, O, \omega)=\sum_{i \in I} \int_{O_{i}} L_{\text {hom }}^{i}\left(\nabla_{\mu} u(x), \omega_{i}\right) d \mu(x)
$$

with $L_{\mathrm{hom}}^{i}: \mathbb{M} \times \Omega_{i} \rightarrow[0, \infty]$ given by

$$
\begin{gathered}
L_{\mathrm{hom}}^{i}\left(\xi, \omega_{i}\right):=\inf _{k \in \mathbb{N}^{*}} \mathbb{E}_{i}^{\mathcal{I}_{i}}\left[\operatorname { i n f } \left\{f_{h_{k}^{i}\left(\stackrel{\cup}{i}_{i}\right)} L^{i}\left(y, \xi+\nabla_{\mu} w(y), \cdot\right) d \mu(y):\right.\right. \\
\left.\left.w \in H_{\mu, 0}^{1, p}\left(h_{k}^{i}\left(\stackrel{\cup}{i}_{i}\right) ; \mathbb{R}^{m}\right)\right\}\right](\omega),
\end{gathered}
$$

where $\mathbb{E}_{i}^{\mathcal{I}_{i}}$ denotes the conditional expectation over $\mathcal{I}_{i}$ with respect to $\mathbb{P}_{i}$, with $\mathcal{I}_{i}$ being the $\sigma$-algebra of invariant sets with respect to $\left(\Omega_{i}, \mathcal{F}_{i}, \mathbb{P}_{i},\left\{\tau_{g}^{i}\right\}_{g \in \mathbb{G}_{i}}\right)$. If in addition $\left(\Omega_{i}, \mathcal{F}_{i}, \mathbb{P}_{i},\left\{\tau_{g}^{i}\right\}_{g \in \mathbb{G}_{i}}\right)$ is ergodic, then $L_{\mathrm{hom}}^{i}$ is deterministic and is given by

$$
\begin{gathered}
L_{\mathrm{hom}}^{i}(\xi):=\inf _{k \in \mathbb{N}^{*}} \mathbb{E}_{i}\left[\operatorname { i n f } \left\{f_{h_{k}^{i}\left(\dot{U}_{i}\right)} L\left(y, \xi+\nabla_{\mu} w(y), \cdot\right) d \mu(y):\right.\right. \\
\left.\left.w \in H_{\mu, 0}^{1, p}\left(h_{k}^{i}\left(\ddot{U}_{i}\right) ; \mathbb{R}^{m}\right)\right\}\right]
\end{gathered}
$$


where $\mathbb{E}_{i}$ denotes the expectation with respect to $\mathbb{P}_{i}$.

Proof of Theorem 3.12. The proof is divided into two steps.

Step 1: using a subadditive result. Let $i \in I$. We prove that for $\mathbb{P}_{i}$-a.e. $\omega_{i} \in \Omega_{i}$, every $x \in O_{i}$ and every $\rho>0$, one has

$$
\varliminf_{n \rightarrow \infty} \mathcal{H}_{\mu}^{\rho} L_{n}\left(x, \xi, \omega_{i}\right)=\varlimsup_{n \rightarrow \infty} \mathcal{H}_{\mu}^{\rho} L_{n}\left(x, \xi, \omega_{i}\right)=L_{\mathrm{hom}}^{i}\left(\xi, \omega_{i}\right) \text { for all } \xi \in \mathbb{M} \text {. }
$$

Let $\xi \in \mathbb{M}$ and let $\mathcal{S}_{i}^{\xi}: \mathcal{B}_{0}^{i} \rightarrow L^{1}\left(\Omega_{i}, \mathcal{F}_{i}, \mathbb{P}_{i}\right)$ be defined by

$$
\mathcal{S}_{i}^{\xi}(A)\left(\omega_{i}\right):=\inf \left\{\int_{\AA} L^{i}\left(y, \xi+\nabla_{\mu} w(y), \omega_{i}\right) d \mu(y): w \in H_{\mu, 0}^{1, p}\left(\AA ; \mathbb{R}^{m}\right)\right\},
$$

where by (3.12) we have $0 \leq \mathcal{S}_{i}^{\xi}(A)\left(\omega_{i}\right) \leq c \mu(\AA) \leq c \mu(A)$ for all $A \in \mathcal{B}_{0}^{i}$ and $\mathbb{P}_{i}$-a.a. $\omega_{i} \in \Omega_{i}$ with $c:=\beta\left(1+|\xi|^{p}\right)$. In particular $\mathcal{S}_{i}^{\xi}$ satisfies the boundedness condition in (2.3). On the other hand, taking (3.13) and (3.14) into account, from $\left(\mathrm{H}_{3}^{i}\right)$, we see that for every $B \in \operatorname{Ba}\left(M_{i} \backslash N_{i}\right)$, every $n \in \mathbb{N}^{*}$ and $\mathbb{P}_{i}$-a.e. $\omega_{i} \in \Omega_{i}$, one has

$$
\begin{aligned}
& \mathcal{S}_{i}^{\xi}\left(h_{n}^{i}(B)\right)\left(\omega_{i}\right)= \inf \left\{\int_{h_{n}^{i}(B)} L^{i}\left(y, \xi+\nabla_{\mu} w(y), \omega_{i}\right) d \mu(y): w \in H_{\mu, 0}^{1, p}\left(h_{n}^{i}(B) ; \mathbb{R}^{m}\right)\right\} \\
&= \inf \left\{\int_{B} L^{i}\left(h_{n}^{i}(y), \xi+\nabla_{\mu} w\left(h_{n}^{i}(y)\right), \omega_{i}\right) d\left(\left(h_{n}^{i}\right)^{-1}\right)^{\sharp} \mu(y):\right. \\
&\left.w \in H_{\mu, 0}^{1, p}\left(h_{n}^{i}(B) ; \mathbb{R}^{m}\right)\right\} \\
&=\mu\left(h_{n}^{i}\left(\mathbb{U}_{i}\right)\right) \inf \left\{\int_{B} L_{n}\left(y, \xi+\nabla_{\mu} w\left(h_{n}^{i}(y)\right), \omega_{i}\right) d \mu(y):\right. \\
&\left.w \in H_{\mu, 0}^{1, p}\left(h_{n}^{i}(B) ; \mathbb{R}^{m}\right)\right\} .
\end{aligned}
$$
But $\mu\left(h_{n}^{i}\left(\mathbb{U}_{i}\right)\right) \mu(B)=\left(\left(h_{n}^{i}\right)^{-1}\right)^{\sharp} \mu(B)=\mu\left(h_{n}^{i}(B)\right)$ by using again $\left(\mathrm{H}_{3}^{i}\right)$, and so from $\left(\mathrm{H}_{5}^{i}\right)$
we obtain

$$
\mathcal{S}_{i}^{\xi}\left(h_{n}^{i}(B)\right)(\omega)=\mu\left(h_{n}^{i}(B)\right) \inf \left\{\int_{B} L_{n}\left(y, \xi+\nabla_{\mu} w(y), \omega_{i}\right) d \mu(y): w \in H_{\mu, 0}^{1, p}\left(B ; \mathbb{R}^{m}\right)\right\}
$$

for all $B \in \operatorname{Ba}\left(M_{i} \backslash N_{i}\right)$, all $n \in \mathbb{N}^{*}$ and $\mathbb{P}_{i}$-a.a. $\omega_{i} \in \Omega_{i}$. Consequently, we have:

$$
\begin{aligned}
\varliminf_{n \rightarrow \infty} \mathcal{H}_{\mu}^{\rho} L_{n}\left(x, \xi, \omega_{i}\right) & =\varliminf_{n \rightarrow \infty} \frac{\mathcal{S}_{i}^{\xi}\left(h_{n}^{i}\left(B_{\rho}(x)\right)\right)\left(\omega_{i}\right)}{\mu\left(h_{n}^{i}\left(B_{\rho}(x)\right)\right.} \\
\varlimsup_{n \rightarrow \infty} \mathcal{H}_{\mu}^{\rho} L_{n}\left(x, \xi, \omega_{i}\right) & =\varlimsup_{n \rightarrow \infty} \frac{\mathcal{S}_{i}^{\xi}\left(h_{n}^{i}\left(B_{\rho}(x)\right)\right)\left(\omega_{i}\right)}{\mu\left(h_{n}^{i}\left(B_{\rho}(x)\right)\right)}
\end{aligned}
$$




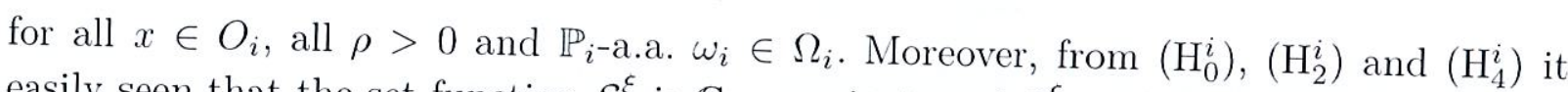
easily seen that the set function $\mathcal{S}_{i}^{\xi}$ is $\mathbb{G}_{i}$-covariant, and $\mathcal{S}_{i}^{\xi}$ is also subadditive because,

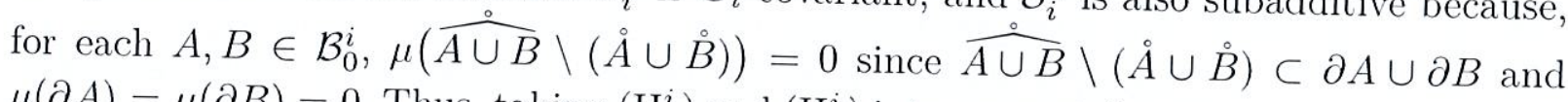
$\mu(\partial A)=\mu(\partial B)=0$. Thus, taking $\left(\mathrm{H}_{1}^{i}\right)$ and $\left(\mathrm{H}_{6}^{i}\right)$ into account, for every $x \in O_{i}$ and every $\rho>0$, we can apply Theorem 2.19 with $\left\{\mathbb{U}_{k}\right\}_{k \in \mathbb{N}^{*}}=\left\{h_{k}^{i}\left(\mathbb{U}_{i}\right)\right\}_{k \in \mathbb{N}^{*}}$ and $\left\{J_{n}\right\}_{n \in \mathbb{N}^{*}}=$ $\left\{h_{n}^{i}\left(B_{\rho}(x)\right)\right\}_{n \in \mathbb{N}^{*}}$, and, noticing that $\mu\left(h_{k}^{i}\left(\mathbb{U}_{i}\right)\right)=\mu\left(\widehat{h_{k}^{i}\left(\mathbb{U}_{i}\right)}\right)=\mu\left(h_{k}^{i}\left(\stackrel{\mathbb{U}}{i}_{i}\right)\right)$ for all $k \in \mathbb{N}^{*}$ (see Remarks (3.10) and (3.11)), we conclude that

$$
\begin{aligned}
\lim _{n \rightarrow \infty} \frac{\mathcal{S}_{i}^{\xi}\left(h_{n}^{i}\left(B_{\rho}(x)\right)\right)\left(\omega_{i}\right)}{\mu\left(h_{n}^{i}\left(B_{\rho}(x)\right)\right)} & =\inf _{k \in \mathbb{N}^{*}} \frac{\mathbb{E}_{i}^{\mathcal{I}_{i}}\left[S_{i}^{\xi}\left(h_{k}^{i}\left(\mathbb{U}_{i}\right)\right)\right]\left(\omega_{i}\right)}{\mu\left(h_{k}^{i}\left(\mathbb{U}_{i}\right)\right)} \\
& =\inf _{k \in \mathbb{N}^{*}} \mathbb{E}_{i}^{\mathcal{I}_{i}}\left[\frac{S_{i}^{\xi}\left(h_{k}^{i}\left(\mathbb{U}_{i}\right)\right)}{\mu\left(h_{k}^{i}\left(\mathbb{U}_{i}\right)\right)}\right]\left(\omega_{i}\right) \\
& =L_{\text {hom }}^{i}\left(\xi, \omega_{i}\right),
\end{aligned}
$$

for $\mathbb{P}_{i}$-a.a. $\omega_{i} \in \Omega_{i}$, and (3.15) follows from (3.16) and (3.17).

Step 2: using a $\Gamma$-convergence result. First of all, as every $L^{i}$ satisfies $(3.12)$ for $\mathbb{P}_{i}$-a.a. $\omega_{i} \in \Omega_{i}$ with $\alpha, \beta>0$ which do depend on $i$, it is easy to see that every $L_{n}$ satisfies (3.5) for $\mathbb{P}$-a.a. $\omega \in \Omega$. So, from Theorem 3.6 it follows that for every $u \in H_{\mu}^{1, p}\left(O ; \mathbb{R}^{m}\right)$ and $\mathbb{P}$-a.e. $\omega \in \Omega$, one has:

(i) $\Gamma\left(L_{\mu}^{p}\right)-\varliminf_{n \rightarrow \infty} E_{n}(u, \cdot, \omega)$ is the restriction to $\mathcal{O}(O)$ of a finite Radon measure which is absolutely continuous with respect to $\mu$;

(ii) $\Gamma\left(L_{\mu}^{p}\right)-\varliminf_{n \rightarrow \infty} E_{n}(u, A, \omega) \geq \int_{A} \varlimsup_{\rho \rightarrow 0} \varliminf_{n \rightarrow \infty} \mathcal{H}_{\mu}^{\rho} L_{n}\left(x, \nabla_{\mu} u(x), \omega\right) d \mu(x)$ for all $A \in \mathcal{O}(O)$;

(iii) $\Gamma\left(L_{\mu}^{p}\right)-\varlimsup_{n \rightarrow \infty} E_{n}(u, O, \omega)=\int_{O} \lim _{\rho \rightarrow 0} \varlimsup_{n \rightarrow \infty} \mathcal{H}_{\mu}^{\rho} L_{n}\left(x, \nabla_{\mu} u(x), \omega\right) d \mu(x)$.

Taking (3.9), (3.10) and (3.11) into account, from (iii) and (3.15) we see that

$$
\begin{aligned}
\Gamma\left(L_{\mu}^{p}\right)-\varlimsup_{n \rightarrow \infty} E_{n}(u, O, \omega) & =\sum_{i \in I} \int_{O_{i}} \lim _{\rho \rightarrow 0} \varlimsup_{n \rightarrow \infty} \mathcal{H}_{\mu}^{\rho} L_{n}\left(x, \nabla_{\mu} u(x), \omega_{i}\right) d \mu(x) \\
& =\sum_{i \in I} \int_{O_{i}} L_{\mathrm{hom}}^{i}\left(\nabla_{\mu} u(x), \omega_{i}\right) d \mu(x) .
\end{aligned}
$$

On the other hand, from (ii) and (3.15) we see that for each $i \in I$, one has

$$
\int_{O_{i}} L_{\mathrm{hom}}^{i}\left(\nabla_{\mu} u(x), \omega_{i}\right) d \mu(x)=\int_{O_{i}} \varlimsup_{\rho \rightarrow 0} \underline{\lim _{n \rightarrow \infty}} \mathcal{H}_{\mu}^{\rho} L_{n}\left(x, \nabla_{\mu} u(x), \omega_{i}\right) d \mu(x)
$$




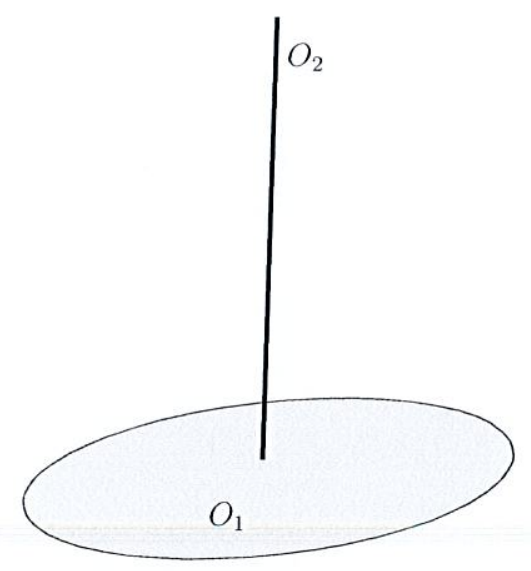

Fig. 1. A plate with a wire.

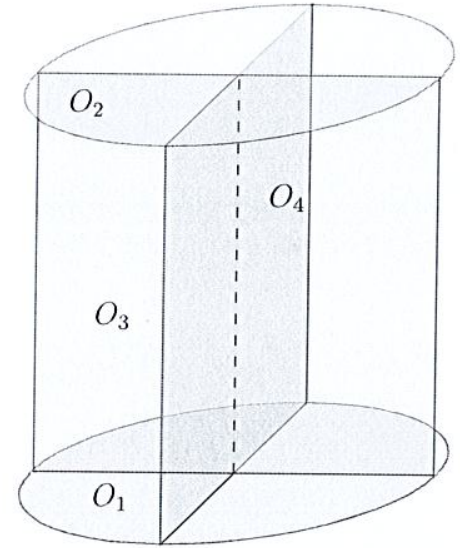

Fig. 2. Two plates connected by intersecting plates.

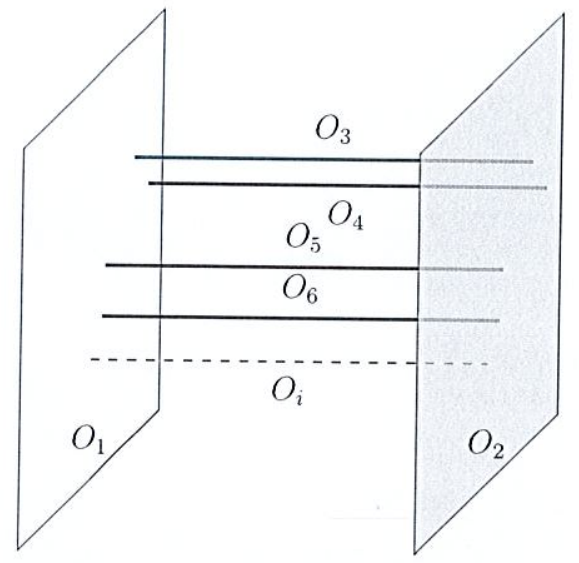

Fig. 3. Two plates connected by wires.

$$
\leq \Gamma\left(L_{\mu}^{p}\right)-\varliminf_{n \rightarrow \infty} E_{n}\left(u, O_{i}, \omega_{i}\right)
$$

Combining (3.18) with (3.19), by using (i) we conclude that

$$
\begin{aligned}
\Gamma\left(L_{\mu}^{p}\right)-\varlimsup_{n \rightarrow \infty} E_{n}(u, O, \omega) & \leq \sum_{i \in I} \Gamma\left(L_{\mu}^{p}\right)-\varliminf_{n \rightarrow \infty} E_{n}\left(u, O_{i}, \omega_{i}\right) \\
& =\Gamma\left(L_{\mu}^{p}\right)-\underline{\lim }_{n \rightarrow \infty} E_{n}(u, O, \omega),
\end{aligned}
$$

and the proof is complete.

The following example gives a simple non-euclidean situation where Theorem 3.12 applies (see Fig. 1). Homogenization of more complex singular structures can be also treated (see Figs. 2 and 3).

Example 3.13. Let $N \geq 1$ and $1 \leq q \leq N-1$ be two integers, let $M:=M_{1} \cup M_{2}$ with:

$$
\begin{aligned}
& M_{1}:=\mathbb{R}^{q} \times\left\{0_{\mathbb{R}^{N-q}}\right\} \\
& M_{2}:=\left\{0_{\mathbb{R}^{q}}\right\} \times \mathbb{R}^{N-q},
\end{aligned}
$$


and let $d_{1}$ (resp. $d_{2}$ ) be the euclidean distance on $M_{1}$ (resp. $M_{2}$ ). Then $d: M \times M \rightarrow$ $[0, \infty[$ defined by

$$
d(x, y):= \begin{cases}d_{1}(x, y) & \text { if } x, y \in M_{1} \\ d_{2}(x, y) & \text { if } x, y \in M_{2} \\ d_{1}\left(x, 0_{\mathbb{R}^{N}}\right)+d_{2}\left(0_{\mathbb{R}^{N}}, y\right) & \text { if } x \in M_{1} \text { and } y \in M_{2} \\ d_{1}\left(0_{\mathbb{R}^{N}}, y\right)+d_{2}\left(x, 0_{\mathbb{R}^{N}}\right) & \text { if } x \in M_{2} \text { and } y \in M_{1}\end{cases}
$$

is a length distance on $M$, and $\mu: \mathcal{B}(M) \rightarrow[0, \infty]$ given by

$$
\mu:=\left.\mathcal{H}^{q}\right|_{M_{1}}+\left.\mathcal{H}^{N-q}\right|_{M_{2}}
$$

is a doubling measure, where $\left.\mathcal{H}^{q}\right|_{M_{1}}$ (resp. $\left.\mathcal{H}^{N-q}\right|_{M_{2}}$ ) denotes the $q$-dimensional (resp. $N-q$-dimensional) Hausdorff measure restricted to $M_{1}$ (resp. $M_{2}$ ). The metric separable complete metric measure space $(M, d, \mu)$ defined above supports a weak $(1, p)$-Poincaré inequality with $p>1$ and satisfies the annular decay property because $(M, d)$ is a length space (see Remark 3.1). Let $\left(\Omega_{1}, \mathcal{F}_{1}, \mathbb{P}_{1}\right)$ and $\left(\Omega_{2}, \mathcal{F}_{2}, \mathbb{P}_{2}\right)$ be two probability spaces and let $(\Omega, \mathcal{F}, \mathbb{P})$ be the product of the probability spaces $\left(\Omega_{1}, \mathcal{F}_{1}, \mathbb{P}_{1}\right)$ and $\left(\Omega_{2}, \mathcal{F}_{2}, \mathbb{P}_{2}\right)$. Let $L: M \times \mathbb{M} \times \Omega \rightarrow[0, \infty]$ be defined by

$$
L(x, \xi, \omega):= \begin{cases}L^{1}\left(x, \xi, \omega_{1}\right) & \text { if } x \in M_{1} \\ L^{2}\left(x, \xi, \omega_{2}\right) & \text { if } x \in M_{2}\end{cases}
$$

where, for $i \in\{1,2\}, L^{i}: M_{i} \times \mathbb{M} \times \Omega_{i} \rightarrow[0, \infty]$ satisfies (3.12) and the following
condition:

$$
L^{i}\left(x+z, \xi, \omega_{i}\right)=L^{i}\left(x, \xi, \tau_{z}^{i}\left(\omega_{i}\right)\right) \text { for all } z \in \mathbb{G}_{i}
$$

with $\mathbb{G}_{1}:=\mathbb{Z}^{q} \times\left\{0_{\mathbb{R}^{N-q}}\right\}$ and $\mathbb{G}_{2}:=\left\{0_{\mathbb{R}^{q}}\right\} \times \mathbb{Z}^{N-q}$, where $\left\{\tau_{z}^{i}\right\}_{z \in \mathbb{G}_{i}}$ is a group of $\mathbb{P}_{i^{-}}$ preserving on $\left(\Omega_{i}, \mathcal{F}_{i}, \mathbb{P}_{i}\right)$. Let $\left\{h_{n}^{1}\right\}_{n \in \mathbb{N}^{*}} \subset \operatorname{Homeo}\left(M_{1}\right)\left(\operatorname{resp} .\left\{h_{n}^{2}\right\}_{n \in \mathbb{N}^{*}} \subset \operatorname{Homeo}\left(M_{2}\right)\right)$ be defined by $h_{n}^{1}(x)=n x\left(\right.$ resp. $\left.h_{n}^{2}(x)=n x\right)$. For each $n \in \mathbb{N}^{*}$, let $L_{n}: M \times \mathbb{M} \times \Omega \rightarrow$ $[0, \infty]$ be given by

$$
\begin{aligned}
L_{n}(x, \xi, \omega) & := \begin{cases}L^{1}\left(h_{n}^{1}(x), \xi, \omega_{1}\right) & \text { if } x \in M_{1} \\
L^{2}\left(h_{n}^{2}(x), \xi, \omega_{2}\right) & \text { if } x \in M_{2},\end{cases} \\
& =L(n x, \xi, \omega) .
\end{aligned}
$$

Let $\mathbb{U}_{1}:=\left[0,1\left[{ }^{q} \times\left\{0_{\mathbb{R}^{N-q}}\right\}\right.\right.$ and $\mathbb{U}_{2}:=\left\{0_{\mathbb{R}^{q}}\right\} \times\left[0,1\left[^{N-q}\right.\right.$ be the unit cells of $M_{1}$ and $M_{2}$ respectively, and let $O:=(]-R, R\left[{ }^{q} \times\left\{0_{\mathbb{R}^{N-q}}\right\}\right) \cup\left(\left\{0_{\mathbb{R}^{q}}\right\} \times\right] 0, R\left[^{N-q}\right)$ where $R>0$. (Here $O_{1}=\left((]-R, 0[\cup] 0, R\left[^{q}\right) \times\left\{0_{\mathbb{R}^{N-q}}\right\}\right)$ and $O_{2}=\left(\left\{0_{\mathbb{R}^{q}}\right\} \times\right] 0, R\left[^{N-q}\right)$.) Then, all the assumptions of Theorem 3.12 are satisfied, and so, for $\mathbb{P}$-a.e. $\omega \in \Omega$, one has 


$$
\begin{aligned}
\Gamma\left(L_{\mu}^{p}\right)-\lim _{n \rightarrow \infty} E_{n}(u, O, \omega)= & \int_{]-R, R\left[^{q}\right.} L_{\mathrm{hom}}^{1}\left(\nabla_{\mu} u(x, 0), \omega_{1}\right) d x \\
& +\int_{] 0, R\left[^{N-q}\right.} L_{\mathrm{hom}}^{2}\left(\nabla_{\mu} u(0, x), \omega_{2}\right) d x
\end{aligned}
$$

for all $u \in H_{\mu}^{1, p}\left(O ; \mathbb{R}^{m}\right)$.

\section{Declaration of competing interest}

There is no competing interest.

\section{References}

[1] Claire Anantharaman, Jean-Philippe Anker, Martine Babillot, Aline Bonami, Bruno Demange, Sandrine Grellier, François Havard, Philippe Jaming, Emmanuel Lesigne, Patrick Maheux, Jean-Pierre Otal, Barbara Schapira, Jean-Pierre Schreiber, Théorèmes ergodiques pour les actions de groupes, Monographies de L'Enseignement Mathématique (Monographs of L'Enseignement Mathématique), vol. 41, L'Enseignement Mathématique, Geneva, 2010. With a foreword in English by Amos Nevo.

[2] Omar Anza Hafsa, Jean-Philippe Mandallena, $\Gamma$-convergence of nonconvex integrals in CheegerSobolev spaces and homogenization, Adv. Calc. Var. 10 (4) (2017) 381-405.

[3] M.A. Akcoglu, U. Krengel, Ergodic theorems for superadditive processes, J. Reine Angew. Math. 323 (1981) 53-67.

[4] Martin R. Bridson, André Haefliger, Metric Spaces of Non-positive Curvature, Grundlehren der Mathematischen Wissenschaften (Fundamental Principles of Mathematical Sciences), vol. 319, Springer-Verlag, Berlin, 1999.

[5] George D. Birkhoff, Proof of the ergodic theorem, Proc. Natl. Acad. Sci. 17 (12) (1931) 656-660.

[6] Stephen M. Buckley, Is the maximal function of a Lipschitz function continuous? Ann. Acad. Sci. Fenn. Math. 24 (2) (1999) 519-528.

[7] A.P. Calderon, A general ergodic theorem, Ann. Math. (2) 58 (1953) 182-191.

[8] J. Cheeger, Differentiability of Lipschitz functions on metric measure spaces, Geom. Funct. Anal. 9 (3) (1999) 428-517.

[9] Tobias H. Colding, William P. Minicozzi II, Liouville theorems for harmonic sections and applications, Commun. Pure Appl. Math. 51 (2) (1998) 113-138.

[10] Yves Derriennic, Sur le théorème ergodique sous-additif, C. R. Acad. Sci. Paris Sér. A-B 281 (22:Aii) (1975) A985-A988.

[11] Mitia Duerinckx, Antoine Gloria, Stochastic homogenization of nonconvex unbounded integral functionals with convex growth, Arch. Ration. Mech. Anal. 221 (3) (2016) 1511-1584.

[12] Anthony H. Dooley, Valentyn Ya. Golodets, Guohua Zhang, Sub-additive ergodic theorems for countable amenable groups, J. Funct. Anal. 267 (5) (2014) 1291-1320.

[13] Gianni Dal Maso, An Introduction to $\Gamma$-Convergence, Progress in Nonlinear Differential Equations and Their Applications, vol. 8, Birkhäuser Boston Inc., Boston, MA, 1993. [14] Gianni Dal Maso, Luciano Modica, Nonlinear stochastic homogenization, Ann. Mat. Pura Appl. (4)
144 (1986) 347-389. [15] Gianni Dal Maso, Luciano Modica, Nonlinear stochastic homogenization and ergodic theory, J.
Reine Angew. Math. 368 (1986) 28-42.

[16] Anthony H. Dooley, Guohua Zhang, Local entropy theory of a random dynamical system, Mem. Am. Math. Soc. 233 (1099) (2015), vi+106.

[17] Frederick P. Greenleaf, Invariant Means on Topological Groups and Their Applications, Van Nostrand Mathematical Studies, vol. 16, Van Nostrand Reinhold Co., New York-Toronto, Ont.-London,
1969. [18] Vladimir Gol'dshtein, Marc Troyanov, Axiomatic theory of Sobolev spaces, Expo. Math. 19 (4)
(2001) 289-336. 
[19] Piotr Hajłasz, Sobolev spaces on metric-measure spaces, in: Heat Kernels and Analysis on Manifolds, Graphs, and Metric Spaces, Paris, 2002, in: Contemp. Math., vol. 338, Amer. Math. Soc., Providence, RI, 2003, pp. 173-218.

[20] Juha Heinonen, Nonsmooth calculus, Bull. Am. Math. Soc. (N. S.) 44 (2) (2007) 163-232.

[21] Juha Heinonen, Pekka Koskela, Quasiconformal maps in metric spaces with controlled geometry, Acta Math. 181 (1) (1998) 1-61.

[22] Juha Heinonen, Pekka Koskela, Nageswari Shanmugalingam, Jeremy T. Tyson, Sobolev Spaces on Metric Measure Spaces, New Mathematical Monographs, vol. 27, Cambridge University Press, Cambridge, 2015. An approach based on upper gradients.

[23] J.M. Hammersley, D.J.A. Welsh, First-passage percolation, subadditive processes, stochastic networks, and generalized renewal theory, in: Proc. Internat. Res. Semin., Statist. Lab., Univ. California, Berkeley, Calif, Springer-Verlag, New York, 1965, pp. 61-110.

[24] Stephen Keith, A differentiable structure for metric measure spaces, Adv. Math. 183 (2) (2004) 271-315.

[25] J.F.C. Kingman, The ergodic theory of subadditive stochastic processes, J. R. Stat. Soc., Ser. B 30 (1968) 499-510.

[26] J.F.C. Kingman, Subadditive ergodic theory, Ann. Probab. 1 (1973) 883-909. With discussion by D.L. Burkholder, Daryl Daley, H. Kesten, P. Ney, Frank Spitzer and J.M. Hammersley, and a reply by the author.

[27] Ulrich Krengel, Ergodic Theorems, De Gruyter Studies in Mathematics, vol. 6, Walter de Gruyter \& Co., Berlin, 1985. With a supplement by Antoine Brunel.

[28] Elon Lindenstrauss, Pointwise theorems for amenable groups, Electron. Res. Announc. Am. Math. Soc. 5 (1999) 82-90.

[29] Elon Lindenstrauss, Pointwise theorems for amenable groups, Invent. Math. 146 (2) (2001) $259-295$.

[30] Christian Licht, Gérard Michaille, Global-local subadditive ergodic theorems and application to homogenization in elasticity, Ann. Math. Blaise Pascal 9 (1) (2002) 21-62.

[31] K. Messaoudi, G. Michaille, Stochastic homogenization of nonconvex integral functionals, RAIRO Modél. Math. Anal. Numér. 28 (3) (1994) 329-356.

[32] Paola Mannucci, Nicolas Dirr, Federica Dragoni, Claudio Marchi, $\gamma$-convergence and homogenisation for a class of degenerate functionals, Nonlinear Anal. (2019), https://doi.org/10.1016/j.na.2019. 111618.

[33] J.v. Neumann, Proof of the quasi-ergodic hypothesis, Proc. Natl. Acad. Sci. 18 (1) (1932) $70-82$.

[34] Xuan-Xanh Nguyen, Ergodic theorems for subadditive spatial processes, Z. Wahrscheinlichkeitstheor. Verw. Geb. 48 (2) (1979) 159-176.

[35] Xuan-Xanh Nguyen, Hans Zessin, Ergodic theorems for spatial processes, Z. Wahrscheinlichkeitstheor. Verw. Geb. 48 (2) (1979) 133-158.

[36] Donald S. Ornstein, Benjamin Weiss, Entropy and isomorphism theorems for actions of amenable groups, J. Anal. Math. 48 (1987) 1-141.

[37] Alan L.T. Paterson, Amenability, Mathematical Surveys and Monographs, vol. 29, American Mathematical Society, Providence, RI, 1988.

[38] Nageswari Shanmugalingam, Newtonian spaces: an extension of Sobolev spaces to metric measure spaces, Rev. Mat. Iberoam. 16 (2) (2000) 243-279.

[39] R.T. Smythe, Multiparameter subadditive processes, Ann. Probab. 4 (5) (1976) 772-782.

[40] A.A. Tempelman, Ergodic theorems for general dynamical systems, Tr. Mosk. Mat. Obŝ. 26 (1972) 95-132.

[41] Arkady Tempelman, Ergodic theorems for group actions, in: Informational and Thermodynamical Aspects, in: Mathematics and Its Applications, vol. 78, Kluwer Academic Publishers Group, Dordrecht, 1992. Translated and revised from the 1986 Russian original. 